CRYSTALLOGRAPHIC COMMUNICATIONS

ISSN 2056-9890

Received 5 June 2018

Accepted 12 June 2018

Edited by D.-J. Xu, Zhejiang University (Yuquan Campus), China

Keywords: platinum(II) complex; hydrido bridge; phosphide ligand; crystal structure.

CCDC reference: 1849017

Supporting information: this article has supporting information at journals.iucr.org/e

\section{Crystal structure of homodinuclear platinum complex containing a metal-metal bond bridged by hydride and phosphide ligands}

\author{
Sakina Ouis, ${ }^{a}$ Djamil Azzedine Rouag, ${ }^{a}$ Lamia Bendjeddou ${ }^{\mathrm{a} *}$ and Corinne Bailly ${ }^{\mathrm{b}}$ \\ aUnité de Recherche Chimie de l'Environnement et Moléculaire, Structurale 'CHEMS', Faculté des Sciences Exactes, \\ Campus Chaabet Ersas, Université Frères Mentouri Constantine 1, 25000 Constantine, Algeria, and ${ }^{\mathbf{b}}$ Service de \\ Radiocristallographie, Institut de Chimie, UMR 7177 CNRS-Université de Strasbourg, 1 Rue Blaise Pascal, 67008 \\ Strasbourg cedex, France. *Correspondence e-mail: bendjeddoulamia@gmail.com
}

In the title compound, $\mu$-diphenylphosphido- $\mu$-hydrido-bis[bromido(triphenylphosphane- $\kappa P)$ platinum(II)] diethyl ether monosolvate, $\left[\mathrm{Pt}_{2} \mathrm{Br}_{2}\left(\mathrm{C}_{12} \mathrm{H}_{10} \mathrm{P}\right) \mathrm{H}\right.$ $\left.\left(\mathrm{C}_{18} \mathrm{H}_{15} \mathrm{P}\right)_{2}\right] \cdot \mathrm{C}_{4} \mathrm{H}_{10} \mathrm{O}$ or $\left[\mathrm{Pt}_{2}(\mu-\mathrm{H})\left(\mu-\mathrm{PPh}_{2}\right) \mathrm{Br}_{2}\left(\mathrm{PPh}_{3}\right)_{2}\right] \cdot\left(\mathrm{C}_{2} \mathrm{H}_{5}\right)_{2} \mathrm{O}$, the $\mathrm{Pt}^{\mathrm{II}}$ atoms are coordinated in a distorted square-planar arrangement, with one hydrido and one phosphido ligand bridging in a trans position. In the lattice, $\mathrm{C}-\mathrm{H} \cdots \mathrm{O}$ and $\mathrm{C}-\mathrm{H} \cdots \pi$ interactions are present. This complex has a total number of 32 electrons, 16 electrons for each $\mathrm{Pt}^{\mathrm{II}}$ atom. One of the $\mathrm{Br}$ atoms is disordered over two positions in a 0.92:0.08 ratio.

\section{Chemical context}

Transition metal hydrides play a central role in many homogeneous catalytic reactions (Bertolasi et al., 1993; Clegg et al., 1996) and are very important in hydrogenation or hydroformylation. Their characterization is commonly carried out by NMR spectroscopy, X-ray analysis or neutron diffraction (Ciriano et al., 1978). Hydrides of $\mathrm{Pt}^{\mathrm{II}}$ are the most numerous of any transition metal hydride group (Leoni et al., 1995; Bachechi et al., 1993). In addition to the presence of the hydride ligand, the complexes invariably have a coordinated phosphine. Pure complexes are usually both air stable and kinetically inert (Roundhill, 1978).

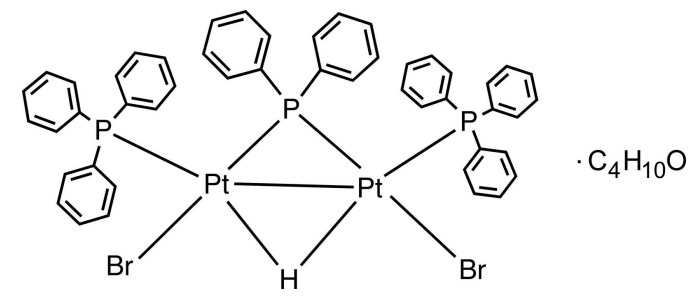

We report here the synthesis and structural analysis of a new hydrido-bridged diplatinum complex, $\mu$-diphenylphosphido- $\mu$-hydrido-bis[bromido(triphenylphosphane- $\kappa P$ )platinum(II)] diethyl ether monosolvate. One of the attractive features of this dinuclear complex is that it is doubly bridged by hydrido and phosphido ligands in a trans fashion. The bridging of metal-metal-bonded homodinuclear complexes with a phosphido ligand allows the stabilization of the metalmetal bond. Many phosphido complexes (with and without a metal-metal bond) have been well documented and both their structural and reactivity features investigated (Stephan, 1989; 
Table 1

Selected geometric parameters $\left(\AA{ }^{\circ}\right)$.

\begin{tabular}{lclc}
\hline Pt1-Pt2 & $2.8365(4)$ & Pt1-P2 & $2.2217(10)$ \\
Pt1-Br1A & $2.5084(6)$ & Pt2-Br2 & $2.5023(5)$ \\
Pt1-Br1B & $2.364(9)$ & Pt2-P2 & $2.2266(11)$ \\
Pt1-P1 & $2.2616(13)$ & Pt2-P3 & $2.2731(11)$ \\
& & & \\
Pt2-Pt1-Br1A & $112.09(2)$ & Pt1-Pt2-P3 & $154.61(3)$ \\
Pt2-Pt1-Br1B & $124.3(3)$ & Br2-Pt2-P2 & $157.93(3)$ \\
Pt2-Pt1-P1 & $155.02(3)$ & Br2-Pt2-P3 & $93.56(3)$ \\
Pt2-Pt1-P2 & $50.46(3)$ & P2-Pt2-P3 & $104.41(4)$ \\
Br1A-Pt1-P1 & $91.84(3)$ & P1-Pt1-H & $169.7(10)$ \\
Br1A-Pt1-P2 & $158.53(3)$ & P2-Pt1-H & $85.5(8)$ \\
Br1B-Pt1-P1 & $80.7(3)$ & Pt2-Pt1-H & $35.1(9)$ \\
Br1B-Pt1-P2 & $174.7(3)$ & Br2-Pt2-H & $77.7(11)$ \\
P1-Pt1-P2 & $104.59(4)$ & P2-Pt2-H & $85.4(11)$ \\
Pt1-Pt2-Br2 & $111.58(1)$ & P3-Pt2-H & $169.6(12)$ \\
Pt1-Pt2-P2 & $50.31(3)$ & Pt1-P2-Pt2 & $79.24(3)$ \\
\hline
\end{tabular}

He et al., 1992; Comte et al., 1997; Lavastre et al., 1997; Mohamed et al., 2015).

\section{Structural commentary}

The asymmetric unit of the title compound consists of an organometallic molecule which is a neutral dinuclear $\mathrm{Pt}^{\mathrm{II}}$ complex, $\left[\mathrm{Pt}_{2}(\mu-\mathrm{H})\left(\mu-\mathrm{PPh}_{2}\right) \mathrm{Br}_{2}\left(\mathrm{PPh}_{3}\right)_{2}\right]$, and one solvent ether molecule (Fig. 1). The dinuclear complex is composed of two triangles formed by two platinum atoms and one phosphorus (P2), and by two platinum and one hydrogen atom. The coordination sphere of each platinum atom is completed by a terminal phosphine (P1, P3) and two bromides $(\mathrm{Br} 1, \mathrm{Br} 2)$. The two platinum atoms are linked by phosphido and hydrido bridges and a formal metal-metal bond. The PtPt bond increases from 2.667 (3) $\AA$ in the starting complex to 2.8365 (4) $\AA$ in the title complex because of the difference in bridging atoms and probably also because of steric hindrance

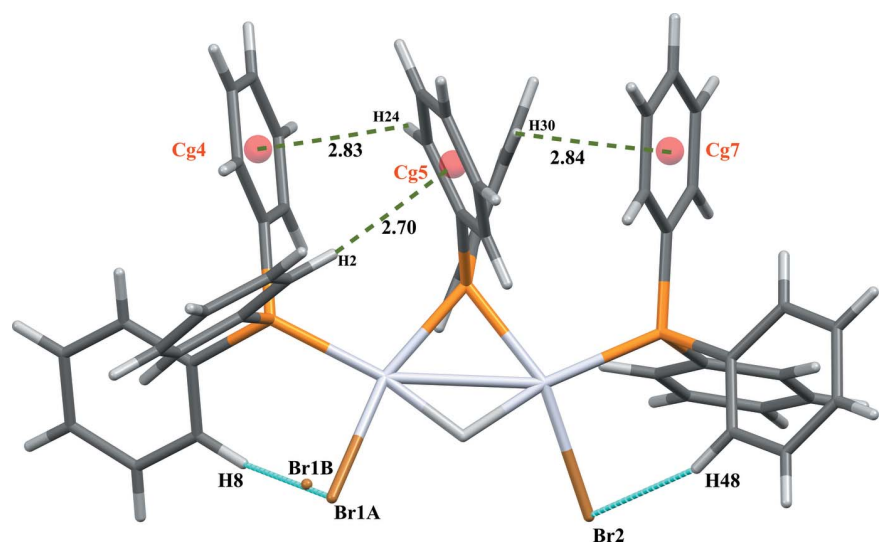

Figure 2

View showing intramolecular $\mathrm{C}-\mathrm{H} \cdots \mathrm{Br}$ hydrogen bonds (blue dashed lines) and $\mathrm{C}-\mathrm{H} \cdots \pi$ (green dashed lines) interactions (Table 2).

between the phenyl groups of the triphenylphosphine unit and repulsion between the triphenylphosphine groups and the bromide atoms. The value of this bond length is comparable to those in dinuclear phosphido- and hydrido-bridged compounds (2.58-2.9 ^; Jans et al., 1983; Bender et al., 1999; Archambault et al., 2001) but is one of the longest. The angle of the phosphido bridge, $\mathrm{Pt} 1-\mathrm{P} 2-\mathrm{Pt} 2$, is $79.24(3)^{\circ}$ because of the long $\mathrm{Pt}-\mathrm{Pt}$ bond and is one of the largest phosphido bridge angles observed (Bachechi et al., 1983). The platinum atoms are coordinated in a distorted square-planar arrangement with one hydrido and one phosphido ligand in a bridging trans position. The phosphorus atoms $\mathrm{P} 1$ and $\mathrm{P} 3$ are located in the same plane as atoms $\mathrm{Pt} 1 / \mathrm{P} 2 / \mathrm{Pt} 2$ with the bromide atoms displaced by 0.0117 (3) and 0.0250 (2) $\AA$ from the plane. The Pt1-P1 and Pt2-P3 bond lengths [2.2616 (13) and 2.2731 (11) $\AA$, respectively] are comparable, and consistent with literature values for a platinum-phosphorus bond. The

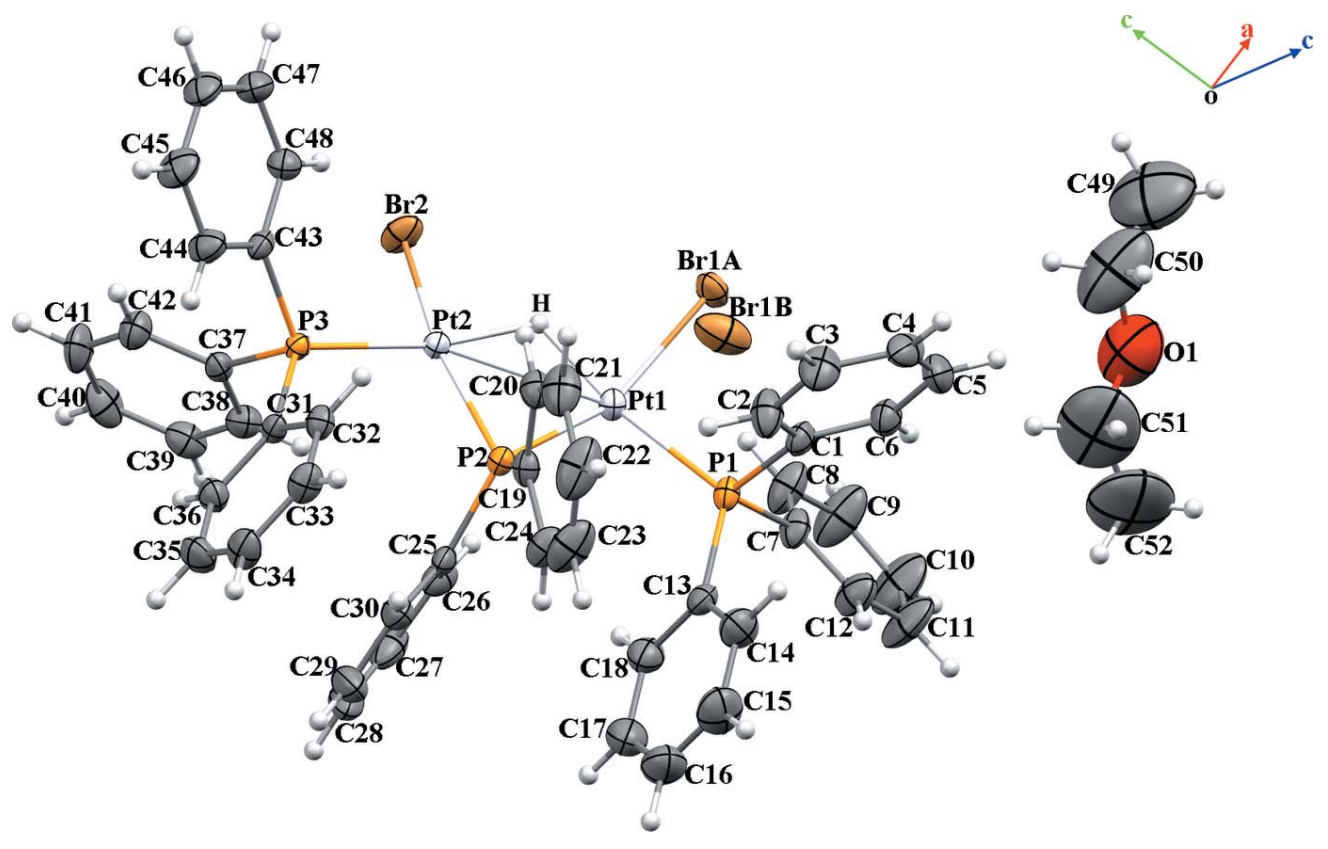

Figure 1

The asymmetric unit of title compound, showing the atom-numbering scheme. Displacement ellipsoids are drawn at the $50 \%$ probability level. 
Table 2

Hydrogen-bond geometry $\left(\AA,^{\circ}\right)$.

$\mathrm{Cg} 4, \mathrm{Cg} 5$ and $\mathrm{Cg} 7$ are the centroids of the C13-C18, C19-C24 and C31-C36 rings, respectively.

\begin{tabular}{lllll}
\hline$D-\mathrm{H} \cdots A$ & $D-\mathrm{H}$ & $\mathrm{H} \cdots A$ & $D \cdots A$ & $D-\mathrm{H} \cdots A$ \\
\hline $\mathrm{C} 8-\mathrm{H} 8 \cdots \mathrm{Br} 1 A$ & 0.95 & 2.73 & $3.460(6)$ & 134 \\
$\mathrm{C} 8-\mathrm{H} 8 \cdots \mathrm{Br} 1 B$ & 0.95 & 1.99 & $2.719(13)$ & 132 \\
$\mathrm{C} 45-\mathrm{H} 45 \cdots \mathrm{O} 1^{\mathrm{i}}$ & 0.95 & 2.54 & $3.484(8)$ & 170 \\
$\mathrm{C} 48-\mathrm{H} 48 \cdots \mathrm{Br} 2$ & 0.95 & 2.65 & $3.520(5)$ & 153 \\
$\mathrm{C} 2-\mathrm{H} 2 \cdots C g 5$ & 0.95 & 2.70 & $3.637(6)$ & 168 \\
$\mathrm{C} 24-\mathrm{H} 24 \cdots C g 4$ & 0.95 & 2.83 & $3.459(9)$ & 125 \\
$\mathrm{C} 30-\mathrm{H} 30 \cdots C g 7$ & 0.95 & 2.84 & $3.340(5)$ & 114 \\
\hline
\end{tabular}

Symmetry code: (i) $x-1, y+1, z$.

$\mathrm{Pt}-\mathrm{Br}$ bonds [2.5023 (5) and 2.5084 (6) $\AA$; Table 1] are slightly longer than those found in the literature (Bachechi et al. 1983). Intramolecular $\mathrm{C}-\mathrm{H} \cdots \mathrm{Br}$ hydrogen bonds and $\mathrm{C}-$ $\mathrm{H} \cdots \pi$ (Table 2, Fig. 2) interactions are observed.

\section{Supramolecular features}

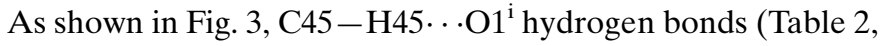
) link the complex and solvent molecules.

\section{NMR Spectroscopy}

The ${ }^{1} \mathrm{H}$ NMR spectrum was recorded on a FT Bruker AVANCE III instrument at $296 \mathrm{~K}$. The spectrum exhibits high field resonance, which unequivocally indicates the presence of a bridging hydride ligand absorbing at $-4.5 \mathrm{ppm}$ (Fig. 4) with ${ }^{1} J \mathrm{Pt}-\mathrm{H} 445 \mathrm{~Hz},{ }^{2} J \mathrm{P} 2-\mathrm{H} 13 \mathrm{~Hz},{ }^{2} J \mathrm{P} 1-\mathrm{H} 87 \mathrm{~Hz}$, identical to ${ }^{2} J$ P3-H (Jans et al., 1983).

\section{Database survey}

A search of the Cambridge Structural Database (Version 5.38; Groom et al., 2016) gave 60 hits for a direct platinum platinum bond with a hydrido bridge. Only one contains the same core as the title compound with hydrido and phosphido bridges (Jans et al., 1983).

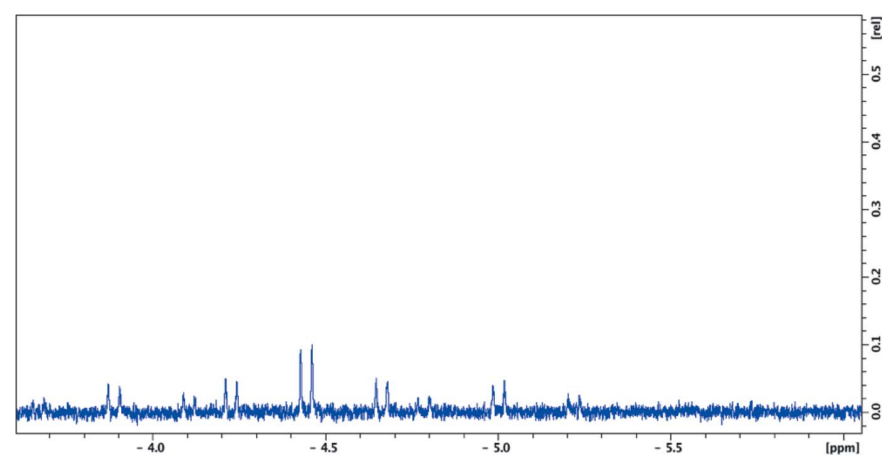

Figure 4

Part of the ${ }^{1} \mathrm{H}$ NMR spectrum of the hydride bridging absorbing at -4.55 ppm.

\section{Synthesis and crystallization}

In a $100 \mathrm{ml}$ schlenk, $\left[\mathrm{Pt}_{2}\left(\mu-o-\mathrm{C}_{6} \mathrm{H}_{4} \mathrm{PPh}_{2}\right)\left(\mathrm{PPh}_{3}\right)_{2}\right](135 \mathrm{mg}$, $0.1 \mathrm{mmol})$ in freshly distilled THF $(30 \mathrm{ml})$ was stirred at room temperature for three $\mathrm{h}$ with a $2 \mathrm{M}$ solution of $\mathrm{HBr}(230 \mu \mathrm{l}$, $0.2 \mathrm{mmol}$ ). The product, a yellow powder, was recrystallized from ether/pentane $(v: v=1: 1)$ yielding pale-yellow crystals.

\section{Refinement}

Crystal data, data collection and structure refinement details are summarized in Table 3 . The hydrido $\mathrm{H}$ atom was located in difference-Fourier maps and refined with a distance restraint of $1.7 \AA$ using the command DFIX in SHELXL2014. C-bound $\mathrm{H}$ atoms were positioned geometrically and refined as riding atoms, with $\mathrm{C}-\mathrm{H}=0.95$ (aromatic), $0.99\left(\mathrm{CH}_{2}\right)$ and $0.98 \AA$ $\left(\mathrm{CH}_{3}\right)$ and with $U_{\text {iso }}(\mathrm{H})=1.2 U_{\text {eq }}(\mathrm{C})$ or $1.5 U_{\text {eq }}($ Cmethyl $)$. Atom $\mathrm{Br} 1$ is disordered over two positions in a 0.92:0.08 ratio.

\section{Funding information}

This work was supported by the Unité de Recherche de Chimie de l'Environnement et Moléculaire Structurale (URCHEMS), Université Frères Mentouri Constantine, Algeria. Thanks are due to MESRS and ATRST (Ministère de

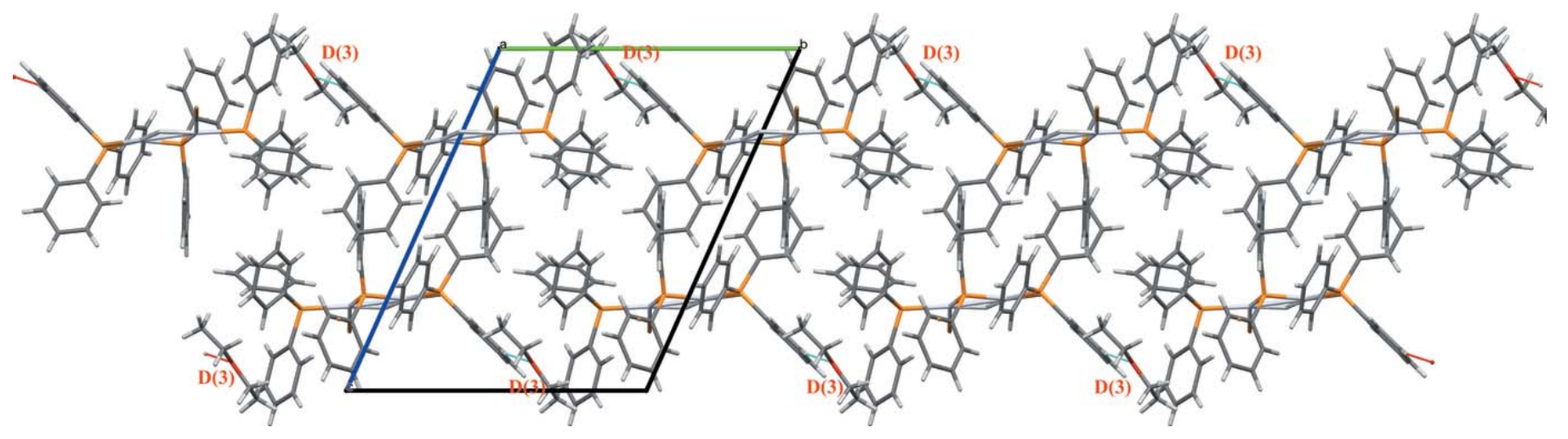

Figure 3

Partial packing diagram of the molecules along the $b$-axis direction, showing the $\mathrm{C}-\mathrm{H} \cdots \mathrm{O}$ hydrogen bonds (Table 2 ). 
l'Enseignement Supérieur et de la Recherche Scientifique) for financial support.

\section{References}

Archambault, C., Bender, R., Braunstein, P., Bouaoud, S. E., Rouag, D., Golhen, S. \& Ouahab, L. (2001). Chem. Commun. pp. 849850.

Bachechi, F., Bracher, G., Grove, D. M., Kellenberger, B., Pregosin, P. S., Venanzi, L. M. \& Zambonelli, L. (1983). Inorg. Chem. 22, 1031-1037.

Bachechi, F., Mura, P. \& Zambonelli, L. (1993). Acta Cryst. C49, 2072-2074.

Bender, R., Bouaoud, S. E., Braunstein, P., Dusausoy, Y., Merabet, N., Raya, J. \& Rouag, D. (1999). J. Chem. Soc. Dalton Trans. pp. 735742.

Bertolasi, V., Ferretti, V., Gilli, P. \& De Benedetti, P. G. (1993). J. Chem. Soc. Perkin Trans. 2, pp. 213-219.

Bruker (2006). APEX2, SADABS and SAINT. Bruker AXS Inc., Madison, Wisconsin, USA.

Ciriano, M., Green, M., Howard, J. A. K., Proud, J. M., Spencer, J. L., Stone, F. G. A. \& Tsipis, C. A. (1978). J. Chem Soc. Dalton Trans. pp. 801

Clegg, W., Capdevila, M., González-Duarte, P. \& Sola, J. (1996). Acta Cryst. B52, 270-276.

Comte, V., Blacque, O., Kubicki, M. M. \& Moïse, C. (1997). Organometallics, 16, 5763-5769.

Dolomanov, O. V., Bourhis, L. J., Gildea, R. J., Howard, J. A. K. \& Puschmann, H. (2009). J. Appl. Cryst. 42, 339-341.

Farrugia, L. J. (2012). J. Appl. Cryst. 45, 849-854.

Groom, C. R., Bruno, I. J., Lightfoot, M. P. \& Ward, S. C. (2016). Acta Cryst. B72, 171-179.

He, Z., Lugan, N., Neibecker, D., Mathieu, R. \& Bonnet, J. J. (1992). J. Organomet. Chem. 426, 247-259.

Jans, J., Naegeli, R., Venanzi, L. M. \& Albinati, A. (1983). J. Organomet. Chem. 247, c37-c41.

Lavastre, O., Bonnet, G., Boni, G., Kubicki, M. M. \& Moïse, C. (1997). J. Organomet. Chem. 547, 141-147.

Leoni, P., Manetti, S. \& Pasquali, M. (1995). Inorg. Chem. 34, 749752.

Macrae, C. F., Edgington, P. R., McCabe, P., Pidcock, E., Shields, G. P., Taylor, R., Towler, M. \& van de Streek, J. (2006). J. Appl. Cryst. 39, 453-457.

Mohamed, A. S., Jourdain, I., Knorr, M., Rousselin, Y. \& Kubicki, M. M. (2015). Acta Cryst. E71, 241-243.
Table 3

Experimental details.

Crystal data

Chemical formula

$M_{\mathrm{r}}$

Crystal system, space group

Temperature (K)

$a, b, c(\AA)$

$\alpha, \beta, \gamma\left({ }^{\circ}\right)$

$V\left(\AA^{3}\right)$

$Z$

Radiation type

$\mu\left(\mathrm{mm}^{-1}\right)$

Crystal size (mm)

$\left[\mathrm{Pt}_{2} \mathrm{Br}_{2}\left(\mathrm{C}_{12} \mathrm{H}_{10} \mathrm{P}\right) \mathrm{H}\left(\mathrm{C}_{18} \mathrm{H}_{15} \mathrm{P}\right)_{2}\right] \cdot$ $\mathrm{C}_{4} \mathrm{H}_{10} \mathrm{O}$

1334.83

Triclinic, $P \overline{1}$

173

11.0257 (5), 14.0341 (6), 17.4469 (7)

113.411 (1), 94.083 (1), 96.681 (1)

2440.09 (18)

2

Mo $K \alpha$

7.50

$0.25 \times 0.16 \times 0.08$

Data collection

Diffractometer

Absorption correction

$T_{\min }, T_{\max }$

No. of measured, independent and observed $[I>2 \sigma(I)]$ reflections

$R_{\text {int }}$
$(\sin \theta / \lambda)_{\max }\left(\AA^{-1}\right)$

Bruker APEXII CCD
Multi-scan ( $S A D A B S$; Bruker,
2006)
$0.512,0.747$
$47421,9969,8359$

0.033
0.625

$0.023,0.057,1.02$
9969
556
23
$\mathrm{H}$ atoms treated by a mixture of
$\quad$ independent and constrained
$\quad$ refinement
$2.00,-1.01$

$\Delta \rho_{\max }, \Delta \rho_{\min }\left(\mathrm{e} \AA^{-3}\right)$

$2.00,-1.01$

Computer programs: APEX2 and SAINT (Bruker, 2006), SHELXS97 and SHELXL97 (Sheldrick, 2008), ORTEP-3 for Windows (Farrugia, 2012), OLEX2 (Dolomanov et al., 2009) and Mercury (Macrae et al., 2006)

Roundhill, D. M. (1978). In Advances in Chemistry, Vol. 167 Transition Metal Hydrides edited by R. Bau, pp. 160-168. Washington: American Chemical Society.

Sheldrick, G. M. (2008). Acta Cryst. A64, 112-122.

Stephan, D. W. (1989). Coord. Chem. Rev. 95, 41-107. 


\section{supporting information}

Acta Cryst. (2018). E74, 977-980］[https://doi.org/10.1107/S205698901800868X]

Crystal structure of homodinuclear platinum complex containing a metal-metal bond bridged by hydride and phosphide ligands

Sakina Ouis, Djamil Azzedine Rouag, Lamia Bendjeddou and Corinne Bailly

Computing details

Data collection: APEX2 (Bruker, 2006); cell refinement: SAINT (Bruker, 2006); data reduction: SAINT (Bruker, 2006); program(s) used to solve structure: SHELXS97 (Sheldrick, 2008); program(s) used to refine structure: SHELXL97 (Sheldrick, 2008); molecular graphics: ORTEP-3 for Windows (Farrugia, 2012); software used to prepare material for publication: OLEX2 (Dolomanov et al., 2009) and Mercury (Macrae et al., 2006).

$\mu$-Diphenylphosphido- $\mu$-hydrido-bis[bromido(triphenylphosphane- $\kappa P$ ) platinum(II)] diethyl ether monosolvate

Crystal data

$\left[\mathrm{Pt}_{2} \mathrm{Br}_{2}\left(\mathrm{C}_{12} \mathrm{H}_{10} \mathrm{P}\right) \mathrm{H}\left(\mathrm{C}_{18} \mathrm{H}_{15} \mathrm{P}\right)_{2}\right] \cdot \mathrm{C}_{4} \mathrm{H}_{10} \mathrm{O}$

$M_{r}=1334.83$

Triclinic, $P \overline{1}$

Hall symbol: -P 1

$a=11.0257(5) \AA$

$b=14.0341(6) \AA$

$c=17.4469(7) \AA$

$\alpha=113.411(1)^{\circ}$

$\beta=94.083(1)^{\circ}$

$\gamma=96.681(1)^{\circ}$

$V=2440.09(18) \AA^{3}$

Data collection

Bruker APEXII CCD

diffractometer

Radiation source: fine-focus sealed tube

Graphite monochromator

Detector resolution: 18.4 pixels $\mathrm{mm}^{-1}$

$\varphi$ and $\omega$ scans

Absorption correction: multi-scan

(SADABS; Bruker, 2006)

$T_{\min }=0.512, T_{\max }=0.747$

Refinement

Refinement on $F^{2}$

Least-squares matrix: full

$R\left[F^{2}>2 \sigma\left(F^{2}\right)\right]=0.023$

$w R\left(F^{2}\right)=0.057$

$S=1.02$

9969 reflections

556 parameters

23 restraints
$Z=2$

$F(000)=1284$

$D_{\mathrm{x}}=1.817 \mathrm{Mg} \mathrm{m}^{-3}$

Mo $K \alpha$ radiation, $\lambda=0.71073 \AA$

Cell parameters from 9992 reflections

$\theta=2.3-35.0^{\circ}$

$\mu=7.50 \mathrm{~mm}^{-1}$

$T=173 \mathrm{~K}$

Plate, yellow

$0.25 \times 0.16 \times 0.08 \mathrm{~mm}$

47421 measured reflections

9969 independent reflections

8359 reflections with $I>2 \sigma(I)$

$R_{\text {int }}=0.033$

$\theta_{\max }=26.4^{\circ}, \theta_{\min }=1.6^{\circ}$

$h=-13 \rightarrow 13$

$k=-17 \rightarrow 17$

$l=-21 \rightarrow 21$

Primary atom site location: structure-invariant direct methods

Hydrogen site location: mixed

$\mathrm{H}$ atoms treated by a mixture of independent and constrained refinement

$w=1 /\left[\sigma^{2}\left(F_{\mathrm{o}}^{2}\right)+(0.0283 P)^{2}+3.5779 P\right]$

where $P=\left(F_{\mathrm{o}}^{2}+2 F_{\mathrm{c}}{ }^{2}\right) / 3$

$(\Delta / \sigma)_{\max }=0.003$ 
$\Delta \rho_{\max }=2.00 \mathrm{e} \AA^{-3}$

$\Delta \rho_{\min }=-1.01$ e $\AA^{-3}$

Special details

Geometry. Bond distances, angles etc. have been calculated using the rounded fractional coordinates. All su's are estimated from the variances of the (full) variance-covariance matrix. The cell esds are taken into account in the estimation of distances, angles and torsion angles

Refinement. Refinement of $\mathrm{F}^{2}$ against ALL reflections. The weighted R-factor $\mathrm{wR}$ and goodness of fit $\mathrm{S}$ are based on $\mathrm{F}^{2}$, conventional $R$-factors $R$ are based on $F$, with $F$ set to zero for negative $F^{2}$. The threshold expression of $F^{2}>2 \operatorname{sigma}\left(F^{2}\right)$ is used only for calculating R-factors(gt) etc. and is not relevant to the choice of reflections for refinement. R-factors based on $\mathrm{F}^{2}$ are statistically about twice as large as those based on $\mathrm{F}$, and $\mathrm{R}$ - factors based on ALL data will be even larger.

Fractional atomic coordinates and isotropic or equivalent isotropic displacement parameters $\left(\AA^{2}\right)$

\begin{tabular}{|c|c|c|c|c|c|}
\hline & $x$ & $y$ & $z$ & $U_{\text {iso }} * / U_{\text {eq }}$ & Occ. $(<1)$ \\
\hline Pt1 & $0.00394(2)$ & $0.88526(2)$ & $0.75388(2)$ & $0.0191(1)$ & \\
\hline $\mathrm{Pt} 2$ & $-0.06369(2)$ & $1.06252(2)$ & $0.73179(2)$ & $0.0177(1)$ & \\
\hline Br1A & $0.21974(5)$ & $0.92152(5)$ & $0.82617(4)$ & $0.0298(2)$ & 0.920 \\
\hline Br1B & $0.2082(8)$ & $0.8680(9)$ & $0.7906(5)$ & $0.054(3)$ & 0.080 \\
\hline $\mathrm{Br} 2$ & $0.11894(4)$ & $1.17492(3)$ & $0.71718(3)$ & $0.0354(1)$ & \\
\hline $\mathrm{P} 1$ & $-0.03201(10)$ & $0.72696(8)$ & $0.76059(7)$ & $0.0225(3)$ & \\
\hline $\mathrm{P} 2$ & $-0.18132(9)$ & $0.91642(7)$ & $0.72085(6)$ & $0.0184(3)$ & \\
\hline P3 & $-0.19935(9)$ & $1.16206(7)$ & $0.70978(6)$ & $0.0173(3)$ & \\
\hline $\mathrm{C} 1$ & $-0.0144(4)$ & $0.7457(3)$ & $0.8704(3)$ & $0.0256(12)$ & \\
\hline $\mathrm{O} 1$ & $0.4696(5)$ & $0.5756(5)$ & $0.9171(4)$ & $0.106(3)$ & \\
\hline $\mathrm{C} 2$ & $-0.0902(5)$ & $0.8066(4)$ & $0.9231(3)$ & $0.0404(17)$ & \\
\hline $\mathrm{C} 3$ & $-0.0777(5)$ & $0.8264(4)$ & $1.0081(3)$ & 0.0439 (19) & \\
\hline $\mathrm{C} 4$ & $0.0081(5)$ & $0.7842(4)$ & $1.0401(3)$ & $0.0373(14)$ & \\
\hline $\mathrm{C} 5$ & $0.0852(5)$ & 0.7255 & $0.9884(3)$ & $0.0388(17)$ & \\
\hline C6 & $0.0748(4)$ & 0.7059 & $0.9034(3)$ & $0.0304(12)$ & \\
\hline $\mathrm{C} 7$ & $0.0683(4)$ & $0.6318(3)$ & $0.7082(3)$ & $0.0300(14)$ & \\
\hline $\mathrm{C} 8$ & $0.1758(5)$ & $0.6624(4)$ & 0.6809 & 0.0457 (17) & \\
\hline C9 & $0.2501(6)$ & $0.5881(5)$ & $0.6409(4)$ & $0.066(2)$ & \\
\hline $\mathrm{C} 10$ & $0.2166(6)$ & $0.4845(5)$ & $0.6282(5)$ & $0.074(3)$ & \\
\hline C11 & $0.1111(6)$ & $0.4548(4)$ & $0.6580(4)$ & $0.064(2)$ & \\
\hline C12 & $0.0373(5)$ & $0.5283(4)$ & $0.6979(4)$ & 0.0444 (19) & \\
\hline C13 & -0.1830 & $0.6475(3)$ & $0.7150(3)$ & $0.0264(12)$ & \\
\hline C14 & $-0.2595(5)$ & $0.6088(4)$ & 0.7591 & 0.0405 (17) & \\
\hline $\mathrm{C} 15$ & $-0.3708(5)$ & $0.5458(4)$ & $0.7194(4)$ & 0.0527 (19) & \\
\hline $\mathrm{C} 16$ & -0.4070 & $0.5213(4)$ & $0.6361(4)$ & 0.0528 (19) & \\
\hline $\mathrm{C} 17$ & $-0.3310(5)$ & $0.5596(4)$ & $0.5912(3)$ & $0.0480(17)$ & \\
\hline C18 & $-0.2199(5)$ & $0.6222(4)$ & $0.6302(3)$ & 0.0357 (14) & \\
\hline C19 & $-0.2826(4)$ & $0.9126(3)$ & 0.7973 & $0.0230(12)$ & \\
\hline $\mathrm{C} 20$ & $-0.2545(4)$ & 0.9915 (3) & 0.8785 & $0.0308(14)$ & \\
\hline $\mathrm{C} 21$ & $-0.3228(5)$ & 0.9889 (4) & 0.9415 & 0.0456 (19) & \\
\hline $\mathrm{C} 22$ & $-0.4174(6)$ & $0.9072(5)$ & $0.9240(4)$ & $0.057(2)$ & \\
\hline $\mathrm{C} 23$ & $-0.4453(5)$ & 0.8285 & $0.8448(4)$ & 0.0523 (19) & \\
\hline $\mathrm{C} 24$ & $-0.3775(4)$ & $0.8291(4)$ & 0.7810 & $0.0342(14)$ & \\
\hline $\mathrm{C} 25$ & $-0.2685(4)$ & $0.8502(3)$ & 0.6165 & $0.0235(11)$ & \\
\hline $\mathrm{C} 26$ & $-0.2012(4)$ & $0.8197(3)$ & $0.5484(3)$ & $0.0313(14)$ & \\
\hline
\end{tabular}




\begin{tabular}{|c|c|c|c|c|}
\hline $\mathrm{C} 27$ & $-0.2604(6)$ & 0.7795 & $0.4669(3)$ & $0.0435(16)$ \\
\hline $\mathrm{C} 28$ & $-0.3867(6)$ & $0.7657(4)$ & 0.4527 & $0.0490(19)$ \\
\hline $\mathrm{C} 29$ & $-0.4536(5)$ & 0.7955 & 0.5183 & $0.0486(17)$ \\
\hline $\mathrm{C} 30$ & -0.3959 (4) & 0.8393 & $0.6011(3)$ & $0.0332(14)$ \\
\hline $\mathrm{C} 31$ & $-0.3612(3)$ & 1.1109 (3) & $0.6999(2)$ & $0.0190(11)$ \\
\hline $\mathrm{C} 32$ & $-0.4044(4)$ & $1.1002(3)$ & 0.7699 (3) & $0.0247(12)$ \\
\hline $\mathrm{C} 33$ & -0.5269 (4) & 1.0618 & $0.7667(3)$ & $0.0298(14)$ \\
\hline $\mathrm{C} 34$ & -0.6077 (4) & $1.0352(4)$ & 0.6945 & $0.0350(16)$ \\
\hline C35 & $-0.5661(4)$ & 1.0467 (4) & $0.6258(3)$ & $0.0358(16)$ \\
\hline $\mathrm{C} 36$ & $-0.4429(4)$ & $1.0840(3)$ & $0.6279(3)$ & $0.0271(12)$ \\
\hline C37 & -0.1800 & $1.1791(3)$ & $0.6131(2)$ & $0.0207(11)$ \\
\hline C38 & -0.1460 & 1.0968 & 0.5462 & $0.0274(12)$ \\
\hline C39 & -0.1318 & $1.1054(4)$ & $0.4710(3)$ & $0.0323(14)$ \\
\hline $\mathrm{C} 40$ & $-0.1494(5)$ & $1.1954(4)$ & $0.4621(3)$ & $0.0428(17)$ \\
\hline $\mathrm{C} 41$ & $-0.1827(5)$ & $1.2783(4)$ & 0.5284 & $0.0441(17)$ \\
\hline $\mathrm{C} 42$ & -0.1988 & $1.2703(3)$ & $0.6036(3)$ & $0.0319(14)$ \\
\hline $\mathrm{C} 43$ & -0.1864 & $1.2956(3)$ & $0.7920(2)$ & $0.0216(11)$ \\
\hline $\mathrm{C} 44$ & $-0.2896(4)$ & $1.3424(3)$ & $0.8157(3)$ & $0.0337(14)$ \\
\hline $\mathrm{C} 45$ & $-0.2768(5)$ & $1.4447(4)$ & $0.8766(3)$ & $0.0400(16)$ \\
\hline $\mathrm{C} 46$ & $-0.1624(5)$ & $1.5005(3)$ & $0.9142(3)$ & $0.0387(16)$ \\
\hline $\mathrm{C} 47$ & $-0.0599(5)$ & $1.4555(3)$ & $0.8906(3)$ & $0.0361(16)$ \\
\hline $\mathrm{C} 48$ & -0.0707 (4) & $1.3532(3)$ & 0.8298 & $0.0284(12)$ \\
\hline C49 & $0.6136(11)$ & $0.7134(8)$ & $1.0225(7)$ & $0.144(5)$ \\
\hline $\mathrm{C} 50$ & $0.4824(10)$ & $0.6726(8)$ & $0.9905(7)$ & $0.127(4)$ \\
\hline $\mathrm{C} 51$ & $0.3504(9)$ & $0.5283(10)$ & $0.8798(9)$ & $0.150(6)$ \\
\hline $\mathrm{C} 52$ & $0.3495(10)$ & $0.4253(9)$ & $0.8167(9)$ & $0.166(6)$ \\
\hline $\mathrm{H}$ & $0.058(2)$ & $1.008(2)$ & $0.757(3)$ & $0.064(17)^{*}$ \\
\hline $\mathrm{H} 2$ & -0.15090 & 0.83502 & 0.90092 & $0.0480^{*}$ \\
\hline $\mathrm{H} 3$ & -0.12886 & 0.86917 & 1.04408 & $0.0530^{*}$ \\
\hline $\mathrm{H} 4$ & 0.01445 & 0.79538 & 1.09771 & $0.0450^{*}$ \\
\hline H5 & 0.14633 & 0.69810 & 1.01109 & $0.0460^{*}$ \\
\hline H6 & 0.12858 & 0.66551 & 0.86829 & $0.0370^{*}$ \\
\hline $\mathrm{H} 8$ & 0.19854 & 0.73368 & 0.68951 & $0.0550^{*}$ \\
\hline H9 & 0.32364 & 0.60878 & 0.62238 & $0.0790^{*}$ \\
\hline H10 & 0.26555 & 0.43337 & 0.59907 & $0.0880^{*}$ \\
\hline H11 & 0.08977 & 0.38394 & 0.65086 & 0.0770 * \\
\hline H12 & -0.03455 & 0.50790 & 0.71826 & $0.0530^{*}$ \\
\hline H14 & -0.23521 & 0.62555 & 0.81695 & $0.0480^{*}$ \\
\hline H15 & -0.42267 & 0.51926 & 0.75006 & $0.0630^{*}$ \\
\hline H16 & -0.48385 & 0.47832 & 0.60909 & $0.0630^{*}$ \\
\hline H17 & -0.35586 & 0.54239 & 0.53344 & 0.0570 * \\
\hline H18 & -0.16815 & 0.64833 & 0.59923 & $0.0420^{*}$ \\
\hline $\mathrm{H} 20$ & -0.18837 & 1.04732 & 0.89073 & 0.0370 * \\
\hline $\mathrm{H} 21$ & -0.30442 & 1.04335 & 0.99663 & $0.0540^{*}$ \\
\hline $\mathrm{H} 22$ & -0.46376 & 0.90550 & 0.96741 & $0.0680^{*}$ \\
\hline $\mathrm{H} 23$ & -0.51153 & 0.77308 & 0.83347 & $0.0620 *$ \\
\hline $\mathrm{H} 24$ & -0.39550 & 0.77317 & 0.72651 & 0.0410 * \\
\hline $\mathrm{H} 26$ & -0.11406 & 0.82651 & 0.55799 & $0.0370 *$ \\
\hline
\end{tabular}




$\begin{array}{lllll}\text { H27 } & -0.21389 & 0.76136 & 0.42076 & 0.0520^{*} \\ \text { H28 } & -0.42742 & 0.73516 & 0.39663 & 0.0590^{*} \\ \text { H29 } & -0.54077 & 0.78634 & 0.50771 & 0.0580^{*} \\ \text { H30 } & -0.44333 & 0.86164 & 0.64663 & 0.0400^{*} \\ \text { H32 } & -0.34940 & 1.11930 & 0.81995 & 0.0300^{*} \\ \text { H33 } & -0.55547 & 1.05384 & 0.81427 & 0.0360^{*} \\ \text { H34 } & -0.69183 & 1.00892 & 0.69241 & 0.0420^{*} \\ \text { H35 } & -0.62203 & 1.02895 & 0.57642 & 0.0430^{*} \\ \text { H36 } & -0.41483 & 1.09099 & 0.57992 & 0.0330^{*} \\ \text { H38 } & -0.13253 & 1.03428 & 0.55205 & 0.0330^{*} \\ \text { H39 } & -0.10969 & 1.04836 & 0.42526 & 0.0390^{*} \\ \text { H40 } & -0.13870 & 1.20118 & 0.41047 & 0.0510^{*} \\ \text { H41 } & -0.19453 & 1.34094 & 0.52218 & 0.0530^{*} \\ \text { H42 } & -0.22263 & 1.32697 & 0.64873 & 0.0390^{*} \\ \text { H44 } & -0.36933 & 1.30437 & 0.79025 & 0.0410^{*} \\ \text { H45 } & -0.34801 & 1.47626 & 0.89230 & 0.0480^{*} \\ \text { H46 } & -0.15438 & 1.57012 & 0.95631 & 0.0460^{*} \\ \text { H47 } & 0.01945 & 1.49446 & 0.91618 & 0.0430^{*} \\ \text { H48 } & 0.00111 & 1.32272 & 0.81398 & 0.0340^{*} \\ \text { H49A } & 0.66194 & 0.69628 & 0.97512 & 0.2160^{*} \\ \text { H49B } & 0.64175 & 0.68122 & 1.05981 & 0.2160^{*} \\ \text { H49C } & 0.62444 & 0.78990 & 1.05398 & 0.2160^{*} \\ \text { H50A } & 0.43940 & 0.66091 & 1.03469 & 0.1520^{*} \\ \text { H50B } & 0.44406 & 0.72478 & 0.97621 & 0.1520^{*} \\ \text { H51A } & 0.31386 & 0.57156 & 0.85407 & 0.1800^{*} \\ \text { H51B } & 0.29961 & 0.52402 & 0.92323 & 0.1800^{*} \\ \text { H52A } & 0.40964 & 0.42814 & 0.77859 & 0.2490^{*} \\ \text { H52B } & 0.26718 & 0.39793 & 0.78456 & 0.2490^{*} \\ \text { H52C } & 0.37106 & 0.37889 & 0.84346 & 0.2490^{*}\end{array}$

Atomic displacement parameters $\left(\AA^{2}\right)$

\begin{tabular}{lllllll}
\hline & $U^{11}$ & $U^{22}$ & $U^{33}$ & $U^{12}$ & $U^{13}$ & $U^{23}$ \\
\hline Pt1 & $0.0181(1)$ & $0.0218(1)$ & $0.0213(1)$ & $0.0054(1)$ & $0.0053(1)$ & $0.0117(1)$ \\
Pt2 & $0.0161(1)$ & $0.0179(1)$ & $0.0205(1)$ & $0.0030(1)$ & $0.0034(1)$ & $0.0090(1)$ \\
Br1A & $0.0185(2)$ & $0.0384(3)$ & $0.0356(4)$ & $0.0010(2)$ & $-0.0020(2)$ & $0.0201(3)$ \\
Br1B & $0.040(4)$ & $0.077(6)$ & $0.038(5)$ & $-0.012(5)$ & $0.004(4)$ & $0.022(4)$ \\
Br2 & $0.0235(2)$ & $0.0294(2)$ & $0.0564(3)$ & $0.0019(2)$ & $0.0144(2)$ & $0.0199(2)$ \\
P1 & $0.0285(6)$ & $0.0227(5)$ & $0.0219(5)$ & $0.0102(4)$ & $0.0067(4)$ & $0.0127(4)$ \\
P2 & $0.0184(5)$ & $0.0174(5)$ & $0.0214(5)$ & $0.0041(4)$ & $0.0044(4)$ & $0.0095(4)$ \\
P3 & $0.0179(5)$ & $0.0155(4)$ & $0.0192(5)$ & $0.0015(4)$ & $0.0027(4)$ & $0.0080(4)$ \\
C1 & $0.035(2)$ & $0.022(2)$ & $0.025(2)$ & $0.0053(17)$ & $0.0061(18)$ & $0.0143(18)$ \\
O1 & $0.085(4)$ & $0.099(4)$ & $0.147(6)$ & $0.030(3)$ & $0.041(4)$ & $0.054(4)$ \\
C2 & $0.058(3)$ & $0.044(3)$ & $0.030(3)$ & $0.026(2)$ & $0.014(2)$ & $0.020(2)$ \\
C3 & $0.066(4)$ & $0.041(3)$ & $0.026(3)$ & $0.019(3)$ & $0.014(2)$ & $0.011(2)$ \\
C4 & $0.057(3)$ & $0.031(2)$ & $0.021(2)$ & $-0.002(2)$ & $-0.001(2)$ & $0.011(2)$ \\
C5 & $0.047(3)$ & $0.038(3)$ & $0.034(3)$ & $0.005(2)$ & $-0.007(2)$ & $0.020(2)$ \\
C6 & $0.035(2)$ & $0.030(2)$ & $0.031(2)$ & $0.0066(19)$ & $0.004(2)$ & $0.017(2)$
\end{tabular}




\begin{tabular}{|c|c|c|c|c|c|c|}
\hline $\mathrm{C} 7$ & $0.040(3)$ & $0.026(2)$ & $0.028(2)$ & $0.0175(19)$ & $0.011(2)$ & 0.0109 (19) \\
\hline $\mathrm{C} 8$ & $0.062(3)$ & $0.036(3)$ & $0.054(3)$ & $0.022(2)$ & $0.032(3)$ & $0.026(3)$ \\
\hline C9 & $0.072(4)$ & $0.048(3)$ & $0.096(5)$ & 0.029 & $0.056(4)$ & $0.036(4)$ \\
\hline $\mathrm{C} 10$ & $0.082(5)$ & $0.043(3)$ & $0.104(6)$ & $0.036(3)$ & $0.051(4)$ & $0.025(4)$ \\
\hline C11 & $0.065(4)$ & $0.027(3)$ & $0.102(5)$ & $0.020(3)$ & $0.031(4)$ & $0.022(3)$ \\
\hline $\mathrm{C} 12$ & $0.037(3)$ & $0.032(3)$ & $0.066(4)$ & $0.012(2)$ & $0.013(3)$ & $0.019(3)$ \\
\hline $\mathrm{C} 13$ & $0.032(2)$ & 0.018 (2) & $0.029(2)$ & $0.0038(17)$ & $0.0044(18)$ & $0.0095(18)$ \\
\hline C14 & $0.040(3)$ & $0.042(3)$ & $0.045(3)$ & $-0.002(2)$ & $0.005(2)$ & $0.026(2)$ \\
\hline $\mathrm{C} 15$ & $0.044(3)$ & $0.056(3)$ & $0.069(4)$ & $-0.002(3)$ & $0.011(3)$ & $0.039(3)$ \\
\hline C16 & $0.041(3)$ & $0.043(3)$ & 0.070 & -0.005 (2) & $-0.006(3)$ & $0.024(3)$ \\
\hline $\mathrm{C} 17$ & 0.057 (3) & 0.039 (3) & $0.041(3)$ & $0.003(2)$ & -0.009 & $0.013(2)$ \\
\hline C18 & $0.045(3)$ & $0.031(2)$ & $0.029(2)$ & $0.005(2)$ & $0.002(2)$ & $0.011(2)$ \\
\hline C19 & $0.021(2)$ & $0.028(2)$ & $0.027(2)$ & $0.0103(16)$ & $0.0091(17)$ & $0.0159(18)$ \\
\hline $\mathrm{C} 20$ & $0.035(3)$ & $0.032(2)$ & $0.029(2)$ & $0.0123(19)$ & $0.006(2)$ & $0.014(2)$ \\
\hline $\mathrm{C} 21$ & $0.062(4)$ & $0.050(3)$ & $0.035(3)$ & $0.030(3)$ & $0.021(3)$ & $0.020(3)$ \\
\hline $\mathrm{C} 22$ & $0.075(4)$ & 0.059 (4) & $0.064(4)$ & $0.034(3)$ & $0.049(3)$ & $0.041(3)$ \\
\hline $\mathrm{C} 23$ & $0.050(3)$ & $0.045(3)$ & 0.077 (4) & $0.011(3)$ & $0.036(3)$ & $0.035(3)$ \\
\hline $\mathrm{C} 24$ & $0.031(2)$ & $0.032(2)$ & $0.046(3)$ & $0.0077(19)$ & $0.016(2)$ & $0.020(2)$ \\
\hline $\mathrm{C} 25$ & $0.033(2)$ & $0.0158(18)$ & $0.024(2)$ & $0.0038(16)$ & $0.0009(17)$ & $0.0110(17)$ \\
\hline $\mathrm{C} 26$ & $0.042(3)$ & $0.023(2)$ & $0.030(2)$ & $0.0040(19)$ & 0.009 (2) & 0.0117 (19) \\
\hline $\mathrm{C} 27$ & $0.082(4)$ & $0.024(2)$ & $0.025(2)$ & $0.012(2)$ & $0.010(3)$ & 0.009 (2) \\
\hline $\mathrm{C} 28$ & $0.077(4)$ & $0.032(3)$ & $0.029(3)$ & $0.015(3)$ & -0.015 & $0.005(2)$ \\
\hline $\mathrm{C} 29$ & 0.049 (3) & 0.039 (3) & 0.047 (3) & 0.009 (2) & -0.019 & 0.010 \\
\hline $\mathrm{C} 30$ & $0.033(2)$ & $0.025(2)$ & $0.037(3)$ & $0.0057(18)$ & $-0.004(2)$ & 0.009 (2) \\
\hline $\mathrm{C} 31$ & $0.0136(18)$ & $0.0187(18)$ & $0.028(2)$ & $0.0033(15)$ & $0.0039(16)$ & $0.0126(17)$ \\
\hline $\mathrm{C} 32$ & $0.027(2)$ & $0.024(2)$ & $0.026(2)$ & $0.0085(17)$ & $0.0064(18)$ & $0.0116(18)$ \\
\hline C33 & $0.026(2)$ & $0.033(2)$ & 0.039 & $0.0084(18)$ & $0.015(2)$ & $0.021(2)$ \\
\hline C34 & $0.020(2)$ & $0.038(3)$ & $0.055(3)$ & $0.0075(19)$ & $0.008(2)$ & $0.026(2)$ \\
\hline $\mathrm{C} 35$ & $0.024(2)$ & $0.043(3)$ & $0.041(3)$ & $0.002(2)$ & $-0.004(2)$ & $0.020(2)$ \\
\hline C36 & $0.024(2)$ & $0.030(2)$ & $0.030(2)$ & $0.0053(17)$ & $0.0010(18)$ & $0.0151(19)$ \\
\hline $\mathrm{C} 37$ & $0.0175(19)$ & $0.025(2)$ & $0.023(2)$ & $0.0005(15)$ & $0.0025(16)$ & $0.0143(17)$ \\
\hline C38 & $0.027(2)$ & $0.029(2)$ & $0.025(2)$ & $0.0015(17)$ & $0.0047(18)$ & 0.0103 \\
\hline C39 & $0.029(2)$ & $0.043(3)$ & $0.023(2)$ & $0.002(2)$ & 0.0065 (18) & $0.012(2)$ \\
\hline $\mathrm{C} 40$ & $0.043(3)$ & $0.066(3)$ & $0.032(3)$ & 0.007 (3) & $0.008(2)$ & $0.033(3)$ \\
\hline $\mathrm{C} 41$ & $0.058(3)$ & $0.047(3)$ & $0.045(3)$ & $0.013(3)$ & 0.007 (3) & $0.036(3)$ \\
\hline $\mathrm{C} 42$ & $0.038(3)$ & $0.030(2)$ & $0.033(2)$ & 0.009 (2) & $0.008(2)$ & $0.017(2)$ \\
\hline $\mathrm{C} 43$ & $0.032(2)$ & $0.0154(18)$ & $0.019(2)$ & $0.0055(16)$ & $0.0052(17)$ & 0.0080 \\
\hline $\mathrm{C} 44$ & $0.032(2)$ & $0.024(2)$ & $0.040(3)$ & $0.0072(19)$ & $0.005(2)$ & 0.007 (2) \\
\hline $\mathrm{C} 45$ & $0.043(3)$ & $0.028(2)$ & $0.046(3)$ & $0.015(2)$ & $0.014(2)$ & $0.008(2)$ \\
\hline $\mathrm{C} 46$ & $0.056(3)$ & $0.021(2)$ & $0.032(3)$ & $0.010(2)$ & $0.002(2)$ & $0.003(2)$ \\
\hline $\mathrm{C} 47$ & $0.043(3)$ & $0.024(2)$ & $0.035(3)$ & $-0.001(2)$ & $-0.005(2)$ & $0.009(2)$ \\
\hline $\mathrm{C} 48$ & $0.031(2)$ & $0.026(2)$ & $0.026(2)$ & $0.0035(18)$ & $0.0010(18)$ & 0.0091 (19) \\
\hline $\mathrm{C} 49$ & $0.157(9)$ & $0.097(7)$ & $0.145(10)$ & $0.033(7)$ & $0.043(8)$ & $0.007(7)$ \\
\hline $\mathrm{C} 50$ & $0.140(7)$ & $0.100(6)$ & $0.170(9)$ & $0.056(6)$ & $0.102(7)$ & $0.062(6)$ \\
\hline $\mathrm{C} 51$ & $0.054(5)$ & $0.175(10)$ & $0.239(14)$ & $0.011(6)$ & $0.044(6)$ & $0.102(9)$ \\
\hline C52 & $0.085(7)$ & $0.139(9)$ & $0.257(15)$ & -0.049 & $-0.013(8)$ & $0.088(9)$ \\
\hline
\end{tabular}


Geometric parameters $\left(\AA,{ }^{\circ}\right)$

\begin{tabular}{|c|c|c|c|}
\hline $\mathrm{Pt} 1-\mathrm{Pt} 2$ & $2.8365(4)$ & $\mathrm{C} 40-\mathrm{C} 41$ & $1.385(7)$ \\
\hline $\mathrm{Pt} 1-\mathrm{Br} 1 \mathrm{~A}$ & $2.5084(6)$ & $\mathrm{C} 41-\mathrm{C} 42$ & $1.382(7)$ \\
\hline $\mathrm{Pt} 1-\mathrm{Br} 1 \mathrm{~B}$ & $2.364(9)$ & $\mathrm{C} 43-\mathrm{C} 48$ & $1.390(6)$ \\
\hline $\mathrm{Pt} 1-\mathrm{P} 1$ & $2.2616(13)$ & $\mathrm{C} 43-\mathrm{C} 44$ & $1.386(6)$ \\
\hline $\mathrm{Pt} 1-\mathrm{P} 2$ & $2.2217(10)$ & $\mathrm{C} 44-\mathrm{C} 45$ & $1.389(7)$ \\
\hline $\mathrm{Pt} 2-\mathrm{Br} 2$ & $2.5023(5)$ & $\mathrm{C} 45-\mathrm{C} 46$ & $1.371(8)$ \\
\hline $\mathrm{Pt} 2-\mathrm{P} 2$ & $2.2266(11)$ & $\mathrm{C} 46-\mathrm{C} 47$ & $1.368(8)$ \\
\hline $\mathrm{Pt} 2-\mathrm{P} 3$ & $2.2731(11)$ & $\mathrm{C} 47-\mathrm{C} 48$ & $1.390(7)$ \\
\hline $\mathrm{Pt} 1-\mathrm{H}$ & $1.73(3)$ & $\mathrm{C} 2-\mathrm{H} 2$ & 0.9500 \\
\hline $\mathrm{Pt} 2-\mathrm{H}$ & $1.73(3)$ & $\mathrm{C} 3-\mathrm{H} 3$ & 0.9500 \\
\hline $\mathrm{P} 1-\mathrm{C} 13$ & $1.823(5)$ & $\mathrm{C} 4-\mathrm{H} 4$ & 0.9500 \\
\hline $\mathrm{P} 1-\mathrm{C} 1$ & $1.820(5)$ & $\mathrm{C} 5-\mathrm{H} 5$ & 0.9500 \\
\hline $\mathrm{P} 1-\mathrm{C} 7$ & $1.833(5)$ & C6-H6 & 0.9500 \\
\hline $\mathrm{P} 2-\mathrm{C} 25$ & $1.817(5)$ & $\mathrm{C} 8-\mathrm{H} 8$ & 0.9500 \\
\hline $\mathrm{P} 2-\mathrm{C} 19$ & $1.812(5)$ & $\mathrm{C} 9-\mathrm{H} 9$ & 0.9500 \\
\hline P3-C37 & $1.819(4)$ & $\mathrm{C} 10-\mathrm{H} 10$ & 0.9500 \\
\hline P3-C31 & 1.814 (4) & $\mathrm{C} 11-\mathrm{H} 11$ & 0.9500 \\
\hline P3-C43 & $1.834(4)$ & $\mathrm{C} 12-\mathrm{H} 12$ & 0.9500 \\
\hline $\mathrm{C} 1-\mathrm{C} 6$ & $1.383(6)$ & $\mathrm{C} 14-\mathrm{H} 14$ & 0.9500 \\
\hline $\mathrm{C} 1-\mathrm{C} 2$ & $1.388(7)$ & C15-H15 & 0.9500 \\
\hline $\mathrm{O} 1-\mathrm{C} 50$ & $1.434(13)$ & $\mathrm{C} 16-\mathrm{H} 16$ & 0.9500 \\
\hline $\mathrm{O} 1-\mathrm{C} 51$ & $1.387(13)$ & C17-H17 & 0.9500 \\
\hline $\mathrm{C} 2-\mathrm{C} 3$ & $1.389(7)$ & C18-H18 & 0.9500 \\
\hline $\mathrm{C} 3-\mathrm{C} 4$ & $1.369(8)$ & $\mathrm{C} 20-\mathrm{H} 20$ & 0.9500 \\
\hline $\mathrm{C} 4-\mathrm{C} 5$ & $1.377(8)$ & $\mathrm{C} 21-\mathrm{H} 21$ & 0.9500 \\
\hline $\mathrm{C} 5-\mathrm{C} 6$ & $1.390(7)$ & $\mathrm{C} 22-\mathrm{H} 22$ & 0.9500 \\
\hline $\mathrm{C} 7-\mathrm{C} 12$ & $1.388(8)$ & $\mathrm{C} 23-\mathrm{H} 23$ & 0.9500 \\
\hline $\mathrm{C} 7-\mathrm{C} 8$ & $1.393(7)$ & $\mathrm{C} 24-\mathrm{H} 24$ & 0.9500 \\
\hline $\mathrm{C} 8-\mathrm{C} 9$ & $1.396(9)$ & $\mathrm{C} 26-\mathrm{H} 26$ & 0.9500 \\
\hline $\mathrm{C} 9-\mathrm{C} 10$ & $1.381(11)$ & $\mathrm{C} 27-\mathrm{H} 27$ & 0.9500 \\
\hline $\mathrm{C} 10-\mathrm{C} 11$ & $1.394(10)$ & $\mathrm{C} 28-\mathrm{H} 28$ & 0.9500 \\
\hline $\mathrm{C} 11-\mathrm{C} 12$ & $1.384(9)$ & $\mathrm{C} 29-\mathrm{H} 29$ & 0.9500 \\
\hline $\mathrm{C} 13-\mathrm{C} 18$ & $1.394(7)$ & $\mathrm{C} 30-\mathrm{H} 30$ & 0.9500 \\
\hline $\mathrm{C} 13-\mathrm{C} 14$ & $1.384(7)$ & $\mathrm{C} 32-\mathrm{H} 32$ & 0.9500 \\
\hline $\mathrm{C} 14-\mathrm{C} 15$ & $1.381(8)$ & $\mathrm{C} 33-\mathrm{H} 33$ & 0.9500 \\
\hline $\mathrm{C} 15-\mathrm{C} 16$ & $1.370(9)$ & C34-H34 & 0.9500 \\
\hline $\mathrm{C} 16-\mathrm{C} 17$ & $1.389(8)$ & C $35-\mathrm{H} 35$ & 0.9500 \\
\hline $\mathrm{C} 17-\mathrm{C} 18$ & $1.375(8)$ & C36-H36 & 0.9500 \\
\hline $\mathrm{C} 19-\mathrm{C} 24$ & $1.399(7)$ & C $38-\mathrm{H} 38$ & 0.9500 \\
\hline $\mathrm{C} 19-\mathrm{C} 20$ & $1.393(7)$ & C39-H39 & 0.9500 \\
\hline $\mathrm{C} 20-\mathrm{C} 21$ & $1.386(7)$ & $\mathrm{C} 40-\mathrm{H} 40$ & 0.9500 \\
\hline $\mathrm{C} 21-\mathrm{C} 22$ & $1.376(9)$ & $\mathrm{C} 41-\mathrm{H} 41$ & 0.9500 \\
\hline $\mathrm{C} 22-\mathrm{C} 23$ & $1.368(9)$ & $\mathrm{C} 42-\mathrm{H} 42$ & 0.9500 \\
\hline $\mathrm{C} 23-\mathrm{C} 24$ & $1.387(8)$ & $\mathrm{C} 44-\mathrm{H} 44$ & 0.9500 \\
\hline $\mathrm{C} 25-\mathrm{C} 26$ & $1.393(7)$ & $\mathrm{C} 45-\mathrm{H} 45$ & 0.9500 \\
\hline $\mathrm{C} 25-\mathrm{C} 30$ & $1.390(6)$ & $\mathrm{C} 46-\mathrm{H} 46$ & 0.9500 \\
\hline
\end{tabular}




\begin{tabular}{|c|c|c|c|}
\hline $\mathrm{C} 26-\mathrm{C} 27$ & $1.382(7)$ & $\mathrm{C} 47-\mathrm{H} 47$ & 0.9500 \\
\hline $\mathrm{C} 27-\mathrm{C} 28$ & $1.374(9)$ & $\mathrm{C} 48-\mathrm{H} 48$ & 0.9500 \\
\hline $\mathrm{C} 28-\mathrm{C} 29$ & $1.357(8)$ & $\mathrm{C} 49-\mathrm{C} 50$ & $1.473(16)$ \\
\hline $\mathrm{C} 29-\mathrm{C} 30$ & $1.394(7)$ & $\mathrm{C} 51-\mathrm{C} 52$ & $1.43(2)$ \\
\hline $\mathrm{C} 31-\mathrm{C} 32$ & $1.398(6)$ & C49-H49A & 0.9800 \\
\hline $\mathrm{C} 31-\mathrm{C} 36$ & $1.384(6)$ & C49-H49B & 0.9800 \\
\hline $\mathrm{C} 32-\mathrm{C} 33$ & $1.383(6)$ & $\mathrm{C} 49-\mathrm{H} 49 \mathrm{C}$ & 0.9800 \\
\hline $\mathrm{C} 33-\mathrm{C} 34$ & $1.382(7)$ & $\mathrm{C} 50-\mathrm{H} 50 \mathrm{~A}$ & 0.9900 \\
\hline C34-C35 & $1.374(7)$ & $\mathrm{C} 50-\mathrm{H} 50 \mathrm{~B}$ & 0.9900 \\
\hline $\mathrm{C} 35-\mathrm{C} 36$ & $1.390(6)$ & C51-H51A & 0.9900 \\
\hline $\mathrm{C} 37-\mathrm{C} 38$ & $1.389(6)$ & $\mathrm{C} 51-\mathrm{H} 51 \mathrm{~B}$ & 0.9900 \\
\hline $\mathrm{C} 37-\mathrm{C} 42$ & $1.391(6)$ & $\mathrm{C} 52-\mathrm{H} 52 \mathrm{~A}$ & 0.9800 \\
\hline C38-C39 & $1.382(7)$ & $\mathrm{C} 52-\mathrm{H} 52 \mathrm{~B}$ & 0.9800 \\
\hline $\mathrm{C} 39-\mathrm{C} 40$ & $1.366(8)$ & $\mathrm{C} 52-\mathrm{H} 52 \mathrm{C}$ & 0.9800 \\
\hline $\mathrm{Pt} 2-\mathrm{Pt} 1-\mathrm{Br} 1 \mathrm{~A}$ & $112.09(2)$ & $\mathrm{C} 43-\mathrm{C} 48-\mathrm{C} 47$ & $120.1(4)$ \\
\hline $\mathrm{Pt} 2-\mathrm{Pt} 1-\mathrm{Br} 1 \mathrm{~B}$ & $124.3(3)$ & $\mathrm{C} 1-\mathrm{C} 2-\mathrm{H} 2$ & 120.00 \\
\hline $\mathrm{Pt} 2-\mathrm{Pt} 1-\mathrm{P} 1$ & $155.02(3)$ & $\mathrm{C} 3-\mathrm{C} 2-\mathrm{H} 2$ & 120.00 \\
\hline $\mathrm{Pt} 2-\mathrm{Pt} 1-\mathrm{P} 2$ & $50.46(3)$ & $\mathrm{C} 4-\mathrm{C} 3-\mathrm{H} 3$ & 120.00 \\
\hline $\mathrm{Br} 1 \mathrm{~A}-\mathrm{Pt} 1-\mathrm{P} 1$ & $91.84(3)$ & $\mathrm{C} 2-\mathrm{C} 3-\mathrm{H} 3$ & 120.00 \\
\hline $\mathrm{Br} 1 \mathrm{~A}-\mathrm{Pt} 1-\mathrm{P} 2$ & $158.53(3)$ & $\mathrm{C} 5-\mathrm{C} 4-\mathrm{H} 4$ & 120.00 \\
\hline $\mathrm{Br} 1 \mathrm{~B}-\mathrm{Pt} 1-\mathrm{P} 1$ & $80.7(3)$ & $\mathrm{C} 3-\mathrm{C} 4-\mathrm{H} 4$ & 120.00 \\
\hline $\mathrm{Br} 1 \mathrm{~B}-\mathrm{Pt} 1-\mathrm{P} 2$ & $174.7(3)$ & $\mathrm{C} 4-\mathrm{C} 5-\mathrm{H} 5$ & 120.00 \\
\hline $\mathrm{P} 1-\mathrm{Pt} 1-\mathrm{P} 2$ & $104.59(4)$ & $\mathrm{C} 6-\mathrm{C} 5-\mathrm{H} 5$ & 120.00 \\
\hline $\mathrm{Pt} 1-\mathrm{Pt} 2-\mathrm{Br} 2$ & $111.58(1)$ & $\mathrm{C} 1-\mathrm{C} 6-\mathrm{H} 6$ & 120.00 \\
\hline $\mathrm{Pt} 1-\mathrm{Pt} 2-\mathrm{P} 2$ & $50.31(3)$ & $\mathrm{C} 5-\mathrm{C} 6-\mathrm{H} 6$ & 120.00 \\
\hline $\mathrm{Pt} 1-\mathrm{Pt} 2-\mathrm{P} 3$ & $154.61(3)$ & $\mathrm{C} 9-\mathrm{C} 8-\mathrm{H} 8$ & 120.00 \\
\hline $\mathrm{Br} 2-\mathrm{Pt} 2-\mathrm{P} 2$ & $157.93(3)$ & $\mathrm{C} 7-\mathrm{C} 8-\mathrm{H} 8$ & 120.00 \\
\hline $\mathrm{Br} 2-\mathrm{Pt} 2-\mathrm{P} 3$ & $93.56(3)$ & $\mathrm{C} 8-\mathrm{C} 9-\mathrm{H} 9$ & 120.00 \\
\hline $\mathrm{P} 2-\mathrm{Pt} 2-\mathrm{P} 3$ & $104.41(4)$ & $\mathrm{C} 10-\mathrm{C} 9-\mathrm{H} 9$ & 120.00 \\
\hline $\mathrm{P} 1-\mathrm{Pt} 1-\mathrm{H}$ & $169.7(10)$ & $\mathrm{C} 11-\mathrm{C} 10-\mathrm{H} 10$ & 120.00 \\
\hline $\mathrm{P} 2-\mathrm{Pt} 1-\mathrm{H}$ & $85.5(8)$ & $\mathrm{C} 9-\mathrm{C} 10-\mathrm{H} 10$ & 120.00 \\
\hline $\mathrm{Br} 1 \mathrm{~A}-\mathrm{Pt} 1-\mathrm{H}$ & $77.9(10)$ & $\mathrm{C} 10-\mathrm{C} 11-\mathrm{H} 11$ & 120.00 \\
\hline $\mathrm{Br} 1 \mathrm{~B}-\mathrm{Pt} 1-\mathrm{H}$ & $89.2(9)$ & $\mathrm{C} 12-\mathrm{C} 11-\mathrm{H} 11$ & 120.00 \\
\hline $\mathrm{Pt} 2-\mathrm{Pt} 1-\mathrm{H}$ & $35.1(9)$ & $\mathrm{C} 11-\mathrm{C} 12-\mathrm{H} 12$ & 120.00 \\
\hline $\mathrm{Pt} 1-\mathrm{Pt} 2-\mathrm{H}$ & $35.1(11)$ & $\mathrm{C} 7-\mathrm{C} 12-\mathrm{H} 12$ & 120.00 \\
\hline $\mathrm{Br} 2-\mathrm{Pt} 2-\mathrm{H}$ & $77.7(11)$ & $\mathrm{C} 15-\mathrm{C} 14-\mathrm{H} 14$ & 120.00 \\
\hline $\mathrm{P} 2-\mathrm{Pt} 2-\mathrm{H}$ & $85.4(11)$ & $\mathrm{C} 13-\mathrm{C} 14-\mathrm{H} 14$ & 120.00 \\
\hline P3—Pt2-H & $169.6(12)$ & $\mathrm{C} 14-\mathrm{C} 15-\mathrm{H} 15$ & 120.00 \\
\hline $\mathrm{Pt} 1-\mathrm{P} 1-\mathrm{C} 1$ & $109.25(16)$ & $\mathrm{C} 16-\mathrm{C} 15-\mathrm{H} 15$ & 120.00 \\
\hline $\mathrm{C} 1-\mathrm{P} 1-\mathrm{C} 7$ & $106.0(2)$ & $\mathrm{C} 17-\mathrm{C} 16-\mathrm{H} 16$ & 120.00 \\
\hline $\mathrm{Pt} 1-\mathrm{P} 1-\mathrm{C} 7$ & $116.75(16)$ & $\mathrm{C} 15-\mathrm{C} 16-\mathrm{H} 16$ & 120.00 \\
\hline $\mathrm{Pt} 1-\mathrm{P} 1-\mathrm{C} 13$ & $117.50(16)$ & $\mathrm{C} 16-\mathrm{C} 17-\mathrm{H} 17$ & 120.00 \\
\hline $\mathrm{C} 7-\mathrm{P} 1-\mathrm{C} 13$ & $100.6(2)$ & $\mathrm{C} 18-\mathrm{C} 17-\mathrm{H} 17$ & 120.00 \\
\hline $\mathrm{C} 1-\mathrm{P} 1-\mathrm{C} 13$ & $105.6(2)$ & $\mathrm{C} 17-\mathrm{C} 18-\mathrm{H} 18$ & 120.00 \\
\hline $\mathrm{Pt} 1-\mathrm{P} 2-\mathrm{Pt} 2$ & $79.24(3)$ & $\mathrm{C} 13-\mathrm{C} 18-\mathrm{H} 18$ & 120.00 \\
\hline $\mathrm{Pt} 1-\mathrm{P} 2-\mathrm{C} 19$ & $110.91(15)$ & $\mathrm{C} 19-\mathrm{C} 20-\mathrm{H} 20$ & 120.00 \\
\hline $\mathrm{Pt} 2-\mathrm{P} 2-\mathrm{C} 19$ & $123.38(16)$ & $\mathrm{C} 21-\mathrm{C} 20-\mathrm{H} 20$ & 120.00 \\
\hline
\end{tabular}




\begin{tabular}{|c|c|c|c|}
\hline $\mathrm{Pt} 2-\mathrm{P} 2-\mathrm{C} 25$ & $110.18(16)$ & $\mathrm{C} 20-\mathrm{C} 21-\mathrm{H} 21$ & 120.00 \\
\hline $\mathrm{Pt} 1-\mathrm{P} 2-\mathrm{C} 25$ & $123.60(15)$ & $\mathrm{C} 22-\mathrm{C} 21-\mathrm{H} 21$ & 120.00 \\
\hline $\mathrm{C} 19-\mathrm{P} 2-\mathrm{C} 25$ & $108.3(2)$ & $\mathrm{C} 23-\mathrm{C} 22-\mathrm{H} 22$ & 120.00 \\
\hline $\mathrm{Pt} 2-\mathrm{P} 3-\mathrm{C} 43$ & $115.63(14)$ & $\mathrm{C} 21-\mathrm{C} 22-\mathrm{H} 22$ & 120.00 \\
\hline $\mathrm{C} 31-\mathrm{P} 3-\mathrm{C} 37$ & $105.31(16)$ & $\mathrm{C} 24-\mathrm{C} 23-\mathrm{H} 23$ & 120.00 \\
\hline $\mathrm{Pt} 2-\mathrm{P} 3-\mathrm{C} 37$ & $110.81(13)$ & $\mathrm{C} 22-\mathrm{C} 23-\mathrm{H} 23$ & 120.00 \\
\hline $\mathrm{C} 37-\mathrm{P} 3-\mathrm{C} 43$ & $105.24(19)$ & $\mathrm{C} 19-\mathrm{C} 24-\mathrm{H} 24$ & 120.00 \\
\hline $\mathrm{C} 31-\mathrm{P} 3-\mathrm{C} 43$ & $102.59(19)$ & $\mathrm{C} 23-\mathrm{C} 24-\mathrm{H} 24$ & 120.00 \\
\hline $\mathrm{Pt} 2-\mathrm{P} 3-\mathrm{C} 31$ & $116.14(15)$ & $\mathrm{C} 25-\mathrm{C} 26-\mathrm{H} 26$ & 120.00 \\
\hline $\mathrm{P} 1-\mathrm{C} 1-\mathrm{C} 2$ & $118.0(4)$ & $\mathrm{C} 27-\mathrm{C} 26-\mathrm{H} 26$ & 120.00 \\
\hline $\mathrm{C} 50-\mathrm{O} 1-\mathrm{C} 51$ & $116.5(8)$ & $\mathrm{C} 28-\mathrm{C} 27-\mathrm{H} 27$ & 120.00 \\
\hline $\mathrm{C} 2-\mathrm{C} 1-\mathrm{C} 6$ & $119.5(4)$ & $\mathrm{C} 26-\mathrm{C} 27-\mathrm{H} 27$ & 120.00 \\
\hline $\mathrm{P} 1-\mathrm{C} 1-\mathrm{C} 6$ & $122.5(4)$ & $\mathrm{C} 27-\mathrm{C} 28-\mathrm{H} 28$ & 120.00 \\
\hline $\mathrm{C} 1-\mathrm{C} 2-\mathrm{C} 3$ & $120.3(5)$ & $\mathrm{C} 29-\mathrm{C} 28-\mathrm{H} 28$ & 120.00 \\
\hline $\mathrm{C} 2-\mathrm{C} 3-\mathrm{C} 4$ & $120.1(5)$ & $\mathrm{C} 30-\mathrm{C} 29-\mathrm{H} 29$ & 120.00 \\
\hline $\mathrm{C} 3-\mathrm{C} 4-\mathrm{C} 5$ & $119.7(5)$ & $\mathrm{C} 28-\mathrm{C} 29-\mathrm{H} 29$ & 120.00 \\
\hline $\mathrm{C} 4-\mathrm{C} 5-\mathrm{C} 6$ & $120.8(5)$ & $\mathrm{C} 25-\mathrm{C} 30-\mathrm{H} 30$ & 120.00 \\
\hline $\mathrm{C} 1-\mathrm{C} 6-\mathrm{C} 5$ & $119.5(4)$ & $\mathrm{C} 29-\mathrm{C} 30-\mathrm{H} 30$ & 120.00 \\
\hline $\mathrm{C} 8-\mathrm{C} 7-\mathrm{C} 12$ & $120.0(5)$ & $\mathrm{C} 33-\mathrm{C} 32-\mathrm{H} 32$ & 120.00 \\
\hline $\mathrm{P} 1-\mathrm{C} 7-\mathrm{C} 12$ & $118.9(4)$ & $\mathrm{C} 31-\mathrm{C} 32-\mathrm{H} 32$ & 120.00 \\
\hline $\mathrm{P} 1-\mathrm{C} 7-\mathrm{C} 8$ & $121.1(4)$ & $\mathrm{C} 34-\mathrm{C} 33-\mathrm{H} 33$ & 120.00 \\
\hline $\mathrm{C} 7-\mathrm{C} 8-\mathrm{C} 9$ & $119.8(6)$ & $\mathrm{C} 32-\mathrm{C} 33-\mathrm{H} 33$ & 120.00 \\
\hline $\mathrm{C} 8-\mathrm{C} 9-\mathrm{C} 10$ & $119.9(6)$ & $\mathrm{C} 33-\mathrm{C} 34-\mathrm{H} 34$ & 120.00 \\
\hline $\mathrm{C} 9-\mathrm{C} 10-\mathrm{C} 11$ & $120.1(6)$ & $\mathrm{C} 35-\mathrm{C} 34-\mathrm{H} 34$ & 120.00 \\
\hline $\mathrm{C} 10-\mathrm{C} 11-\mathrm{C} 12$ & $120.1(6)$ & $\mathrm{C} 34-\mathrm{C} 35-\mathrm{H} 35$ & 120.00 \\
\hline $\mathrm{C} 7-\mathrm{C} 12-\mathrm{C} 11$ & $120.0(5)$ & $\mathrm{C} 36-\mathrm{C} 35-\mathrm{H} 35$ & 120.00 \\
\hline $\mathrm{P} 1-\mathrm{C} 13-\mathrm{C} 14$ & $123.6(4)$ & $\mathrm{C} 35-\mathrm{C} 36-\mathrm{H} 36$ & 120.00 \\
\hline $\mathrm{P} 1-\mathrm{C} 13-\mathrm{C} 18$ & $117.2(4)$ & $\mathrm{C} 31-\mathrm{C} 36-\mathrm{H} 36$ & 120.00 \\
\hline $\mathrm{C} 14-\mathrm{C} 13-\mathrm{C} 18$ & $119.2(5)$ & $\mathrm{C} 37-\mathrm{C} 38-\mathrm{H} 38$ & 120.00 \\
\hline $\mathrm{C} 13-\mathrm{C} 14-\mathrm{C} 15$ & $120.3(5)$ & $\mathrm{C} 39-\mathrm{C} 38-\mathrm{H} 38$ & 120.00 \\
\hline $\mathrm{C} 14-\mathrm{C} 15-\mathrm{C} 16$ & $120.4(5)$ & $\mathrm{C} 40-\mathrm{C} 39-\mathrm{H} 39$ & 120.00 \\
\hline $\mathrm{C} 15-\mathrm{C} 16-\mathrm{C} 17$ & $119.8(5)$ & $\mathrm{C} 38-\mathrm{C} 39-\mathrm{H} 39$ & 120.00 \\
\hline $\mathrm{C} 16-\mathrm{C} 17-\mathrm{C} 18$ & $120.2(5)$ & $\mathrm{C} 39-\mathrm{C} 40-\mathrm{H} 40$ & 120.00 \\
\hline $\mathrm{C} 13-\mathrm{C} 18-\mathrm{C} 17$ & $120.2(5)$ & $\mathrm{C} 41-\mathrm{C} 40-\mathrm{H} 40$ & 120.00 \\
\hline $\mathrm{P} 2-\mathrm{C} 19-\mathrm{C} 20$ & $117.3(3)$ & $\mathrm{C} 42-\mathrm{C} 41-\mathrm{H} 41$ & 120.00 \\
\hline $\mathrm{C} 20-\mathrm{C} 19-\mathrm{C} 24$ & $119.2(4)$ & $\mathrm{C} 40-\mathrm{C} 41-\mathrm{H} 41$ & 120.00 \\
\hline $\mathrm{P} 2-\mathrm{C} 19-\mathrm{C} 24$ & $123.1(4)$ & $\mathrm{C} 37-\mathrm{C} 42-\mathrm{H} 42$ & 120.00 \\
\hline $\mathrm{C} 19-\mathrm{C} 20-\mathrm{C} 21$ & $120.2(4)$ & $\mathrm{C} 41-\mathrm{C} 42-\mathrm{H} 42$ & 120.00 \\
\hline $\mathrm{C} 20-\mathrm{C} 21-\mathrm{C} 22$ & $119.8(5)$ & $\mathrm{C} 45-\mathrm{C} 44-\mathrm{H} 44$ & 120.00 \\
\hline $\mathrm{C} 21-\mathrm{C} 22-\mathrm{C} 23$ & $120.7(6)$ & $\mathrm{C} 43-\mathrm{C} 44-\mathrm{H} 44$ & 120.00 \\
\hline $\mathrm{C} 22-\mathrm{C} 23-\mathrm{C} 24$ & $120.4(6)$ & $\mathrm{C} 44-\mathrm{C} 45-\mathrm{H} 45$ & 120.00 \\
\hline $\mathrm{C} 19-\mathrm{C} 24-\mathrm{C} 23$ & $119.6(5)$ & $\mathrm{C} 46-\mathrm{C} 45-\mathrm{H} 45$ & 120.00 \\
\hline $\mathrm{P} 2-\mathrm{C} 25-\mathrm{C} 26$ & $116.9(3)$ & $\mathrm{C} 47-\mathrm{C} 46-\mathrm{H} 46$ & 120.00 \\
\hline $\mathrm{P} 2-\mathrm{C} 25-\mathrm{C} 30$ & $123.7(4)$ & $\mathrm{C} 45-\mathrm{C} 46-\mathrm{H} 46$ & 120.00 \\
\hline $\mathrm{C} 26-\mathrm{C} 25-\mathrm{C} 30$ & $118.9(4)$ & $\mathrm{C} 46-\mathrm{C} 47-\mathrm{H} 47$ & 120.00 \\
\hline $\mathrm{C} 25-\mathrm{C} 26-\mathrm{C} 27$ & $120.4(5)$ & $\mathrm{C} 48-\mathrm{C} 47-\mathrm{H} 47$ & 120.00 \\
\hline $\mathrm{C} 26-\mathrm{C} 27-\mathrm{C} 28$ & $119.9(5)$ & $\mathrm{C} 43-\mathrm{C} 48-\mathrm{H} 48$ & 120.00 \\
\hline $\mathrm{C} 27-\mathrm{C} 28-\mathrm{C} 29$ & $120.4(5)$ & $\mathrm{C} 47-\mathrm{C} 48-\mathrm{H} 48$ & 120.00 \\
\hline
\end{tabular}


$\mathrm{C} 28-\mathrm{C} 29-\mathrm{C} 30$
$\mathrm{C} 25-\mathrm{C} 30-\mathrm{C} 29$
$\mathrm{C} 32-\mathrm{C} 31-\mathrm{C} 36$
$\mathrm{P} 3-\mathrm{C} 31-\mathrm{C} 36$
$\mathrm{P} 3-\mathrm{C} 31-\mathrm{C} 32$
$\mathrm{C} 31-\mathrm{C} 32-\mathrm{C} 33$
$\mathrm{C} 32-\mathrm{C} 33-\mathrm{C} 34$
$\mathrm{C} 33-\mathrm{C} 34-\mathrm{C} 35$
$\mathrm{C} 34-\mathrm{C} 35-\mathrm{C} 36$
$\mathrm{C} 31-\mathrm{C} 36-\mathrm{C} 35$
$\mathrm{P} 3-\mathrm{C} 37-\mathrm{C} 38$
$\mathrm{C} 38-\mathrm{C} 37-\mathrm{C} 42$
$\mathrm{P} 3-\mathrm{C} 37-\mathrm{C} 42$
$\mathrm{C} 37-\mathrm{C} 38-\mathrm{C} 39$
$\mathrm{C} 38-\mathrm{C} 39-\mathrm{C} 40$
C39-C $40-\mathrm{C} 41$
C40-C $41-\mathrm{C} 42$
C37-C $42-\mathrm{C} 41$
C44-C $43-\mathrm{C} 48$
P3-C $43-\mathrm{C} 44$
P3-C43-C48
C43-C $44-\mathrm{C} 45$
C44-C $45-\mathrm{C} 46$
C45-C $46-\mathrm{C} 47$
C46-C $47-\mathrm{C} 48$

$\mathrm{Br} 1 \mathrm{~A}-\mathrm{Pt} 1-\mathrm{Pt} 2-\mathrm{Br} 2$

$\mathrm{Br} 1 \mathrm{~A}-\mathrm{Pt} 1-\mathrm{Pt} 2-\mathrm{P} 2$

$\mathrm{Br} 1 \mathrm{~A}-\mathrm{Pt} 1-\mathrm{Pt} 2-\mathrm{P} 3$

$\mathrm{P} 1-\mathrm{Pt} 1-\mathrm{Pt} 2-\mathrm{Br} 2$

$\mathrm{P} 1-\mathrm{Pt} 1-\mathrm{Pt} 2-\mathrm{P} 2$

$\mathrm{P} 1-\mathrm{Pt} 1-\mathrm{Pt} 2-\mathrm{P} 3$

$\mathrm{P} 2-\mathrm{Pt} 1-\mathrm{Pt} 2-\mathrm{Br} 2$

$\mathrm{P} 2-\mathrm{Pt} 1-\mathrm{Pt} 2-\mathrm{P} 3$

$\mathrm{Pt} 2-\mathrm{Pt} 1-\mathrm{P} 1-\mathrm{C} 1$

$\mathrm{Br} 1 \mathrm{~A}-\mathrm{Pt} 1-\mathrm{P} 1-\mathrm{C} 1$

$\mathrm{P} 2-\mathrm{Pt} 1-\mathrm{P} 1-\mathrm{C} 1$

$\mathrm{Pt} 2-\mathrm{Pt} 1-\mathrm{P} 1-\mathrm{C} 7$

$\mathrm{Br} 1 \mathrm{~A}-\mathrm{Pt} 1-\mathrm{P} 1-\mathrm{C} 7$

$\mathrm{P} 2-\mathrm{Pt} 1-\mathrm{P} 1-\mathrm{C} 7$

$\mathrm{Pt} 2-\mathrm{Pt} 1-\mathrm{P} 1-\mathrm{C} 13$

$\mathrm{Br} 1 \mathrm{~A}-\mathrm{Pt} 1-\mathrm{P} 1-\mathrm{C} 13$

$\mathrm{P} 2-\mathrm{Pt1}-\mathrm{P} 1-\mathrm{C} 13$

$\mathrm{Br} 1 \mathrm{~A}-\mathrm{Pt} 1-\mathrm{P} 2-\mathrm{Pt} 2$

$\mathrm{P} 1-\mathrm{Pt} 1-\mathrm{P} 2-\mathrm{Pt} 2$

Pt2-Pt1-P2-C19

Br1A-Pt1-P2-C19

$\mathrm{P} 1-\mathrm{Pt} 1-\mathrm{P} 2-\mathrm{C} 19$
$120.8(5)$

119.5 (4)

$119.1(4)$

$123.5(3)$

$117.3(3)$

$120.3(4)$

$120.1(4)$

$119.9(4)$

$120.6(4)$

$120.0(4)$

$118.1(3)$

$119.2(4)$

122.7 (3)

120.3 (4)

$120.4(5)$

$120.0(5)$

$120.3(5)$

$119.9(4)$

$118.8(4)$

$121.5(3)$

119.7 (3)

120.2 (4)

$120.6(5)$

$119.7(5)$

$120.7(5)$

29.49 (3)

$-165.31(4)$

$-159.11(7)$

$-168.15(7)$

$-2.95(8)$

$3.25(11)$

$-165.20(4)$

$6.20(7)$

$-103.09(17)$

60.59 (16)

$-105.44(16)$

$136.76(17)$

$-59.55(17)$

$134.41(17)$

17.2 (2)

$-179.16(18)$

$14.81(18)$

$39.94(10)$

$178.72(4)$

$-121.79(17)$

$-81.85(19)$

$56.92(17)$
$\mathrm{O} 1-\mathrm{C} 50-\mathrm{C} 49$

$\mathrm{O} 1-\mathrm{C} 51-\mathrm{C} 52$

$\mathrm{C} 50-\mathrm{C} 49-\mathrm{H} 49 \mathrm{~A}$

C $50-\mathrm{C} 49-\mathrm{H} 49 \mathrm{~B}$

C $50-\mathrm{C} 49-\mathrm{H} 49 \mathrm{C}$

$\mathrm{H} 49 \mathrm{~A}-\mathrm{C} 49-\mathrm{H} 49 \mathrm{~B}$

$\mathrm{H} 49 \mathrm{~A}-\mathrm{C} 49-\mathrm{H} 49 \mathrm{C}$

$\mathrm{H} 49 \mathrm{~B}-\mathrm{C} 49-\mathrm{H} 49 \mathrm{C}$

$\mathrm{O} 1-\mathrm{C} 50-\mathrm{H} 50 \mathrm{~A}$

$\mathrm{O} 1-\mathrm{C} 50-\mathrm{H} 50 \mathrm{~B}$

C49- $\mathrm{C} 50-\mathrm{H} 50 \mathrm{~A}$

$\mathrm{C} 49-\mathrm{C} 50-\mathrm{H} 50 \mathrm{~B}$

$\mathrm{H} 50 \mathrm{~A}-\mathrm{C} 50-\mathrm{H} 50 \mathrm{~B}$

$\mathrm{O} 1-\mathrm{C} 51-\mathrm{H} 51 \mathrm{~A}$

$\mathrm{O} 1-\mathrm{C} 51-\mathrm{H} 51 \mathrm{~B}$

C52-C51-H51A

C52-C51-H51B

$\mathrm{H} 51 \mathrm{~A}-\mathrm{C} 51-\mathrm{H} 51 \mathrm{~B}$

C $51-\mathrm{C} 52-\mathrm{H} 52 \mathrm{~A}$

C $51-\mathrm{C} 52-\mathrm{H} 52 \mathrm{~B}$

$\mathrm{C} 51-\mathrm{C} 52-\mathrm{H} 52 \mathrm{C}$

$\mathrm{H} 52 \mathrm{~A}-\mathrm{C} 52-\mathrm{H} 52 \mathrm{~B}$

$\mathrm{H} 52 \mathrm{~A}-\mathrm{C} 52-\mathrm{H} 52 \mathrm{C}$

$\mathrm{H} 52 \mathrm{~B}-\mathrm{C} 52-\mathrm{H} 52 \mathrm{C}$

$\mathrm{C} 43-\mathrm{P} 3-\mathrm{C} 31-\mathrm{C} 32$

$\mathrm{C} 31-\mathrm{P} 3-\mathrm{C} 37-\mathrm{C} 42$

$\mathrm{C} 37-\mathrm{P} 3-\mathrm{C} 31-\mathrm{C} 36$

$\mathrm{C} 31-\mathrm{P} 3-\mathrm{C} 43-\mathrm{C} 44$

$\mathrm{C} 43-\mathrm{P} 3-\mathrm{C} 37-\mathrm{C} 38$

$\mathrm{Pt} 2-\mathrm{P} 3-\mathrm{C} 37-\mathrm{C} 42$

$\mathrm{Pt} 2-\mathrm{P} 3-\mathrm{C} 43-\mathrm{C} 48$

$\mathrm{C} 43-\mathrm{P} 3-\mathrm{C} 31-\mathrm{C} 36$

$\mathrm{C} 43-\mathrm{P} 3-\mathrm{C} 37-\mathrm{C} 42$

$\mathrm{C} 37-\mathrm{P} 3-\mathrm{C} 43-\mathrm{C} 48$

$\mathrm{Pt} 2-\mathrm{P} 3-\mathrm{C} 31-\mathrm{C} 36$

$\mathrm{C} 31-\mathrm{P} 3-\mathrm{C} 43-\mathrm{C} 48$

$\mathrm{C} 50-\mathrm{O} 1-\mathrm{C} 51-\mathrm{C} 52$

$\mathrm{C} 2-\mathrm{C} 1-\mathrm{C} 6-\mathrm{C} 5$

$\mathrm{C} 51-\mathrm{O} 1-\mathrm{C} 50-\mathrm{C} 49$

$\mathrm{C} 6-\mathrm{C} 1-\mathrm{C} 2-\mathrm{C} 3$

$\mathrm{P} 1-\mathrm{C} 1-\mathrm{C} 2-\mathrm{C} 3$

$\mathrm{P} 1-\mathrm{C} 1-\mathrm{C} 6-\mathrm{C} 5$

$\mathrm{C} 1-\mathrm{C} 2-\mathrm{C} 3-\mathrm{C} 4$

$\mathrm{C} 2-\mathrm{C} 3-\mathrm{C} 4-\mathrm{C} 5$

$\mathrm{C} 3-\mathrm{C} 4-\mathrm{C} 5-\mathrm{C} 6$

$\mathrm{C} 4-\mathrm{C} 5-\mathrm{C} 6-\mathrm{C} 1$
$110.2(9)$

110.7 (9)

110.00

109.00

109.00

109.00

109.00

109.00

110.00

110.00

110.00

110.00

108.00

109.00

110.00

109.00

109.00

108.00

109.00

110.00

110.00

109.00

109.00

109.00

$-63.9(4)$

87.1 (4)

$5.2(4)$

$-15.4(4)$

$159.6(3)$

$-146.6(3)$

$39.4(4)$

115.1 (4)

-20.9 (4)

-83.3 (4)

$-117.8(4)$

$166.8(3)$

$-170.5(10)$

1.5 (7)

$-179.7(10)$

-0.8 (8)

$-177.8(4)$

178.3 (4)

$-1.2(9)$

$2.6(9)$

$-1.9(9)$

$-0.2(8)$ 


\begin{tabular}{|c|c|c|c|}
\hline $\mathrm{Pt} 2-\mathrm{Pt} 1-\mathrm{P} 2-\mathrm{C} 25$ & $107.17(19)$ & $\mathrm{C} 8-\mathrm{C} 7-\mathrm{C} 12-\mathrm{C} 11$ & $-2.1(8)$ \\
\hline $\mathrm{Br} 1 \mathrm{~A}-\mathrm{Pt} 1-\mathrm{P} 2-\mathrm{C} 25$ & $147.11(19)$ & $\mathrm{P} 1-\mathrm{C} 7-\mathrm{C} 8-\mathrm{C} 9$ & $-179.7(4)$ \\
\hline $\mathrm{P} 1-\mathrm{Pt} 1-\mathrm{P} 2-\mathrm{C} 25$ & $-74.11(19)$ & $\mathrm{P} 1-\mathrm{C} 7-\mathrm{C} 12-\mathrm{C} 11$ & $179.5(5)$ \\
\hline $\mathrm{Br} 2-\mathrm{Pt} 2-\mathrm{P} 2-\mathrm{Pt} 1$ & $39.20(9)$ & $\mathrm{C} 12-\mathrm{C} 7-\mathrm{C} 8-\mathrm{C} 9$ & $2.0(8)$ \\
\hline $\mathrm{P} 3-\mathrm{Pt} 2-\mathrm{P} 2-\mathrm{Pt} 1$ & $-177.26(3)$ & $\mathrm{C} 7-\mathrm{C} 8-\mathrm{C} 9-\mathrm{C} 10$ & $0.3(9)$ \\
\hline $\mathrm{Pt} 1-\mathrm{Pt} 2-\mathrm{P} 2-\mathrm{C} 19$ & $108.05(19)$ & $\mathrm{C} 8-\mathrm{C} 9-\mathrm{C} 10-\mathrm{C} 11$ & $-2.3(10)$ \\
\hline $\mathrm{Br} 2-\mathrm{Pt} 2-\mathrm{P} 2-\mathrm{C} 19$ & $147.25(18)$ & $\mathrm{C} 9-\mathrm{C} 10-\mathrm{C} 11-\mathrm{C} 12$ & $2.2(11)$ \\
\hline $\mathrm{P} 3-\mathrm{Pt} 2-\mathrm{P} 2-\mathrm{C} 19$ & $-69.21(18)$ & $\mathrm{C} 10-\mathrm{C} 11-\mathrm{C} 12-\mathrm{C} 7$ & $0.1(10)$ \\
\hline $\mathrm{Pt} 1-\mathrm{Pt} 2-\mathrm{P} 2-\mathrm{C} 25$ & $-122.02(16)$ & $\mathrm{P} 1-\mathrm{C} 13-\mathrm{C} 14-\mathrm{C} 15$ & $-177.6(4)$ \\
\hline $\mathrm{Br} 2-\mathrm{P} t 2-\mathrm{P} 2-\mathrm{C} 25$ & $-82.82(18)$ & $\mathrm{C} 14-\mathrm{C} 13-\mathrm{C} 18-\mathrm{C} 17$ & $0.0(8)$ \\
\hline $\mathrm{P} 3-\mathrm{Pt} 2-\mathrm{P} 2-\mathrm{C} 25$ & $60.72(16)$ & $\mathrm{C} 18-\mathrm{C} 13-\mathrm{C} 14-\mathrm{C} 15$ & $0.1(8)$ \\
\hline $\mathrm{Pt} 1-\mathrm{Pt} 2-\mathrm{P} 3-\mathrm{C} 31$ & $0.85(15)$ & $\mathrm{P} 1-\mathrm{C} 13-\mathrm{C} 18-\mathrm{C} 17$ & $177.9(4)$ \\
\hline $\mathrm{Br} 2-\mathrm{Pt} 2-\mathrm{P} 3-\mathrm{C} 31$ & $172.84(12)$ & $\mathrm{C} 13-\mathrm{C} 14-\mathrm{C} 15-\mathrm{C} 16$ & $-0.3(9)$ \\
\hline $\mathrm{P} 2-\mathrm{Pt} 2-\mathrm{P} 3-\mathrm{C} 31$ & $5.77(13)$ & $\mathrm{C} 14-\mathrm{C} 15-\mathrm{C} 16-\mathrm{C} 17$ & $0.3(9)$ \\
\hline $\mathrm{Pt} 1-\mathrm{Pt} 2-\mathrm{P} 3-\mathrm{C} 37$ & $-119.22(14)$ & $\mathrm{C} 15-\mathrm{C} 16-\mathrm{C} 17-\mathrm{C} 18$ & $-0.2(9)$ \\
\hline $\mathrm{Br} 2-\mathrm{Pt} 2-\mathrm{P} 3-\mathrm{C} 37$ & $52.77(14)$ & $\mathrm{C} 16-\mathrm{C} 17-\mathrm{C} 18-\mathrm{C} 13$ & $0.0(9)$ \\
\hline $\mathrm{P} 2-\mathrm{Pt} 2-\mathrm{P} 3-\mathrm{C} 37$ & $-114.30(14)$ & $\mathrm{P} 2-\mathrm{C} 19-\mathrm{C} 20-\mathrm{C} 21$ & $-175.3(4)$ \\
\hline $\mathrm{Pt} 1-\mathrm{Pt} 2-\mathrm{P} 3-\mathrm{C} 43$ & $121.17(16)$ & $\mathrm{C} 20-\mathrm{C} 19-\mathrm{C} 24-\mathrm{C} 23$ & $2.7(7)$ \\
\hline $\mathrm{Br} 2-\mathrm{Pt} 2-\mathrm{P} 3-\mathrm{C} 43$ & $-66.84(15)$ & $\mathrm{P} 2-\mathrm{C} 19-\mathrm{C} 24-\mathrm{C} 23$ & $175.4(4)$ \\
\hline $\mathrm{P} 2-\mathrm{Pt} 2-\mathrm{P} 3-\mathrm{C} 43$ & $126.09(15)$ & $\mathrm{C} 24-\mathrm{C} 19-\mathrm{C} 20-\mathrm{C} 21$ & $-2.2(7)$ \\
\hline $\mathrm{Pt} 1-\mathrm{P} 1-\mathrm{C} 1-\mathrm{C} 2$ & $59.8(4)$ & $\mathrm{C} 19-\mathrm{C} 20-\mathrm{C} 21-\mathrm{C} 22$ & $1.0(8)$ \\
\hline $\mathrm{C} 7-\mathrm{P} 1-\mathrm{C} 1-\mathrm{C} 2$ & $-173.7(4)$ & $\mathrm{C} 20-\mathrm{C} 21-\mathrm{C} 22-\mathrm{C} 23$ & $-0.3(10)$ \\
\hline $\mathrm{C} 13-\mathrm{P} 1-\mathrm{C} 1-\mathrm{C} 2$ & $-67.5(4)$ & $\mathrm{C} 21-\mathrm{C} 22-\mathrm{C} 23-\mathrm{C} 24$ & $0.8(10)$ \\
\hline $\mathrm{Pt} 1-\mathrm{P} 1-\mathrm{C} 1-\mathrm{C} 6$ & $-117.1(4)$ & $\mathrm{C} 22-\mathrm{C} 23-\mathrm{C} 24-\mathrm{C} 19$ & $-2.1(9)$ \\
\hline $\mathrm{C} 7-\mathrm{P} 1-\mathrm{C} 1-\mathrm{C} 6$ & $9.5(5)$ & $\mathrm{C} 26-\mathrm{C} 25-\mathrm{C} 30-\mathrm{C} 29$ & $1.9(7)$ \\
\hline $\mathrm{C} 13-\mathrm{P} 1-\mathrm{C} 1-\mathrm{C} 6$ & $115.6(4)$ & $\mathrm{C} 30-\mathrm{C} 25-\mathrm{C} 26-\mathrm{C} 27$ & $0.2(7)$ \\
\hline $\mathrm{Pt} 1-\mathrm{P} 1-\mathrm{C} 13-\mathrm{C} 14$ & $-124.8(4)$ & $\mathrm{P} 2-\mathrm{C} 25-\mathrm{C} 26-\mathrm{C} 27$ & $-172.8(4)$ \\
\hline $\mathrm{Pt} 1-\mathrm{P} 1-\mathrm{C} 7-\mathrm{C} 8$ & $12.7(5)$ & $\mathrm{P} 2-\mathrm{C} 25-\mathrm{C} 30-\mathrm{C} 29$ & $174.3(4)$ \\
\hline $\mathrm{C} 1-\mathrm{P} 1-\mathrm{C} 7-\mathrm{C} 8$ & $-109.2(4)$ & $\mathrm{C} 25-\mathrm{C} 26-\mathrm{C} 27-\mathrm{C} 28$ & $-2.5(7)$ \\
\hline $\mathrm{C} 13-\mathrm{P} 1-\mathrm{C} 7-\mathrm{C} 8$ & $141.0(4)$ & $\mathrm{C} 26-\mathrm{C} 27-\mathrm{C} 28-\mathrm{C} 29$ & $2.9(8)$ \\
\hline $\mathrm{Pt} 1-\mathrm{P} 1-\mathrm{C} 7-\mathrm{C} 12$ & $-169.1(4)$ & $\mathrm{C} 27-\mathrm{C} 28-\mathrm{C} 29-\mathrm{C} 30$ & $-0.8(9)$ \\
\hline $\mathrm{C} 1-\mathrm{P} 1-\mathrm{C} 7-\mathrm{C} 12$ & $69.1(5)$ & $\mathrm{C} 28-\mathrm{C} 29-\mathrm{C} 30-\mathrm{C} 25$ & $-1.5(8)$ \\
\hline $\mathrm{C} 13-\mathrm{P} 1-\mathrm{C} 7-\mathrm{C} 12$ & $-40.7(5)$ & $\mathrm{C} 36-\mathrm{C} 31-\mathrm{C} 32-\mathrm{C} 33$ & $0.9(7)$ \\
\hline $\mathrm{C} 7-\mathrm{P} 1-\mathrm{C} 13-\mathrm{C} 14$ & $107.3(5)$ & $\mathrm{P} 3-\mathrm{C} 31-\mathrm{C} 32-\mathrm{C} 33$ & $179.9(4)$ \\
\hline $\mathrm{Pt} 1-\mathrm{P} 1-\mathrm{C} 13-\mathrm{C} 18$ & $57.4(4)$ & $\mathrm{C} 32-\mathrm{C} 31-\mathrm{C} 36-\mathrm{C} 35$ & $-0.1(7)$ \\
\hline $\mathrm{C} 1-\mathrm{P} 1-\mathrm{C} 13-\mathrm{C} 14$ & $-2.7(5)$ & $\mathrm{P} 3-\mathrm{C} 31-\mathrm{C} 36-\mathrm{C} 35$ & $-179.1(4)$ \\
\hline $\mathrm{C} 7-\mathrm{P} 1-\mathrm{C} 13-\mathrm{C} 18$ & $-70.4(4)$ & $\mathrm{C} 31-\mathrm{C} 32-\mathrm{C} 33-\mathrm{C} 34$ & $-0.9(7)$ \\
\hline $\mathrm{C} 1-\mathrm{P} 1-\mathrm{C} 13-\mathrm{C} 18$ & $179.6(4)$ & $\mathrm{C} 32-\mathrm{C} 33-\mathrm{C} 34-\mathrm{C} 35$ & $0.2(8)$ \\
\hline $\mathrm{C} 25-\mathrm{P} 2-\mathrm{C} 19-\mathrm{C} 20$ & $-154.3(4)$ & $\mathrm{C} 33-\mathrm{C} 34-\mathrm{C} 35-\mathrm{C} 36$ & $0.6(8)$ \\
\hline $\mathrm{C} 19-\mathrm{P} 2-\mathrm{C} 25-\mathrm{C} 26$ & $-163.2(4)$ & $\mathrm{C} 34-\mathrm{C} 35-\mathrm{C} 36-\mathrm{C} 31$ & $-0.6(8)$ \\
\hline $\mathrm{Pt} 1-\mathrm{P} 2-\mathrm{C} 25-\mathrm{C} 30$ & $156.3(3)$ & $\mathrm{P} 3-\mathrm{C} 37-\mathrm{C} 38-\mathrm{C} 39$ & $179.1(3)$ \\
\hline $\mathrm{Pt} 1-\mathrm{P} 2-\mathrm{C} 25-\mathrm{C} 26$ & $-31.1(4)$ & $\mathrm{C} 42-\mathrm{C} 37-\mathrm{C} 38-\mathrm{C} 39$ & $-0.4(6)$ \\
\hline $\mathrm{Pt} 1-\mathrm{P} 2-\mathrm{C} 19-\mathrm{C} 20$ & $67.2(4)$ & $\mathrm{C} 38-\mathrm{C} 37-\mathrm{C} 42-\mathrm{C} 41$ & $-0.5(6)$ \\
\hline $\mathrm{Pt} 2-\mathrm{P} 2-\mathrm{C} 19-\mathrm{C} 20$ & $-23.6(4)$ & $\mathrm{P} 3-\mathrm{C} 37-\mathrm{C} 42-\mathrm{C} 41$ & $-179.9(4)$ \\
\hline $\mathrm{Pt} 2-\mathrm{P} 2-\mathrm{C} 25-\mathrm{C} 26$ & $59.2(4)$ & $\mathrm{C} 37-\mathrm{C} 38-\mathrm{C} 39-\mathrm{C} 40$ & $0.9(7)$ \\
\hline $\mathrm{Pt} 1-\mathrm{P} 2-\mathrm{C} 19-\mathrm{C} 24$ & $-105.6(4)$ & $\mathrm{C} 38-\mathrm{C} 39-\mathrm{C} 40-\mathrm{C} 41$ & $-0.6(8)$ \\
\hline $\mathrm{Pt} 2-\mathrm{P} 2-\mathrm{C} 19-\mathrm{C} 24$ & $163.7(3)$ & $\mathrm{C} 39-\mathrm{C} 40-\mathrm{C} 41-\mathrm{C} 42$ & $-0.3(8)$ \\
\hline $\mathrm{P} 2-\mathrm{P} 2-\mathrm{C} 25-\mathrm{C} 30$ & $-113.5(4)$ & $\mathrm{C} 40-\mathrm{C} 41-\mathrm{C} 42-\mathrm{C} 37$ & $0.8(7)$ \\
\hline
\end{tabular}




$\begin{array}{llll}\mathrm{C} 25-\mathrm{P} 2-\mathrm{C} 19-\mathrm{C} 24 & 33.0(5) & \mathrm{P} 3-\mathrm{C} 43-\mathrm{C} 44-\mathrm{C} 45 & -178.3(4) \\ \mathrm{C} 19-\mathrm{P} 2-\mathrm{C} 25-\mathrm{C} 30 & 24.2(5) & \mathrm{C} 44-\mathrm{C} 43-\mathrm{C} 48-\mathrm{C} 47 & 0.5(7) \\ \mathrm{P} 12-\mathrm{P} 3-\mathrm{C} 31-\mathrm{C} 32 & 63.2(4) & \mathrm{C} 48-\mathrm{C} 43-\mathrm{C} 44-\mathrm{C} 45 & -0.5(7) \\ \mathrm{C} 37-\mathrm{P} 3-\mathrm{C} 31-\mathrm{C} 32 & -173.8(3) & \mathrm{P} 3-\mathrm{C} 43-\mathrm{C} 48-\mathrm{C} 47 & 178.3(4) \\ \mathrm{C} 37-\mathrm{P} 3-\mathrm{C} 43-\mathrm{C} 44 & 94.5(4) & \mathrm{C} 43-\mathrm{C} 44-\mathrm{C} 45-\mathrm{C} 46 & -0.2(8) \\ \mathrm{P} 2-\mathrm{P} 3-\mathrm{C} 43-\mathrm{C} 44 & -142.9(3) & \mathrm{C} 44-\mathrm{C} 45-\mathrm{C} 46-\mathrm{C} 47 & 1.0(8) \\ \mathrm{Pt} 2-\mathrm{P} 3-\mathrm{C} 37-\mathrm{C} 38 & 34.0(3) & \mathrm{C} 45-\mathrm{C} 46-\mathrm{C} 47-\mathrm{C} 48 & -1.0(7) \\ \mathrm{C} 31-\mathrm{P} 3-\mathrm{C} 37-\mathrm{C} 38 & -92.4(3) & \mathrm{C} 46-\mathrm{C} 47-\mathrm{C} 48-\mathrm{C} 43 & 0.3(7)\end{array}$

Hydrogen-bond geometry $\left(A,{ }^{\circ}\right)$

$\mathrm{Cg} 4, \mathrm{Cg} 5$ and $\mathrm{Cg} 7$ are the centroids of the $\mathrm{C} 13-\mathrm{C} 18, \mathrm{C} 19-\mathrm{C} 24$ and $\mathrm{C} 31-\mathrm{C} 36$ rings, respectively.

\begin{tabular}{lllll}
\hline$D-\mathrm{H} \cdots A$ & $D-\mathrm{H}$ & $\mathrm{H} \cdots A$ & $D \cdots A$ & $D-\mathrm{H} \cdots A$ \\
\hline $\mathrm{C} 8-\mathrm{H} 8 \cdots \mathrm{Br} 1 A$ & 0.95 & 2.73 & $3.460(6)$ & 134 \\
$\mathrm{C} 8-\mathrm{H} 8 \cdots \mathrm{Br} 1 B$ & 0.95 & 1.99 & $2.719(13)$ & 132 \\
$\mathrm{C} 45-\mathrm{H} 45 \cdots \mathrm{O} 1^{\mathrm{i}}$ & 0.95 & 2.54 & $3.484(8)$ & 170 \\
$\mathrm{C} 48-\mathrm{H} 48 \cdots \mathrm{Br} 2$ & 0.95 & 2.65 & $3.520(5)$ & 153 \\
$\mathrm{C} 2-\mathrm{H} 2 \cdots C g 5$ & 0.95 & 2.70 & $3.637(6)$ & 168 \\
$\mathrm{C} 24-\mathrm{H} 24 \cdots C g 4$ & 0.95 & 2.83 & $3.459(9)$ & 125 \\
$\mathrm{C} 30-\mathrm{H} 30 \cdots C g 7$ & 0.95 & 2.84 & $3.340(5)$ & 114
\end{tabular}

Symmetry code: (i) $x-1, y+1, z$. 